\title{
Mathematical modeling of the smoking dynamics using frac- tional differential equations with local and nonlocal kernel
}

\author{
V. F. Morales-Delgadoa , J. F. Gómez-Aguilarb,*, M. A. Taneco-Hernández ${ }^{\mathrm{a}}$, R. F. Escobar-Jiménezc, V. H. \\ Olivares-Peregrino ${ }^{\mathrm{C}}$ \\ ${ }^{a}$ Facultad de Matemáticas, Universidad Autónoma de Guerrero. Av. Lázaro Cárdenas S/N, Cd. Universitaria. Chilpancingo, Guerrero, \\ México. \\ ${ }^{\text {b} C O N A C y T-T e c n o l o ́ g i c o ~ N a c i o n a l ~ d e ~ M e ́ x i c o / C E N I D E T, ~ I n t e r i o r ~ I n t e r n a d o ~ P a l m i r a ~ S / N, ~ C o l . ~ P a l m i r a, ~ C . P . ~ 62490, ~ C u e r n a v a c a, ~ M o r e l o s, ~}$ \\ México. \\ ${ }^{c}$ Tecnológico Nacional de México/CENIDET, Interior Internado Palmira S/N, Col. Palmira, C.P. 62490, Cuernavaca, Morelos, México.
}

\author{
Communicated by A. Atangana
}

\begin{abstract}
In this paper, we analyze the fractional modeling of the giving up the smoking using the definitions of Liouville-Caputo and Atangana-Baleanu-Caputo fractional derivatives. Applying the homotopy analysis method and the Laplace transform with polynomial homotopy, the analytical solution of the smoking dynamics has obtained. Furthermore, using an iterative scheme by the Laplace transform, and the Atangana-Baleanu fractional integral, special solutions of the model are obtained. Uniqueness and existence of the solutions by the fixed-point theorem and Picard-Lindelof approach are studied. Finally, some numerical simulations are carried out for illustrating the results obtained.
\end{abstract}

Keywords: Smoking model, Liouville-Caputo fractional derivative, Atangana-Baleanu fractional derivative, Laplace transform, homotopy method.

2010 MSC: 92C50, 26A33, 44A10, 65H20.

(C)2018 All rights reserved.

\section{Introduction}

Smoking is one of the main causes of health problems in the world. According to the world health organization tobacco smoking is the prodigious cause of the cancer, heart disease, stomach ulcers, asthma, stained teeth, high blood pressure, vascular diseases and chronic obstructive lung diseases among others. All these harmful diseases are caused because a cigarette contains thousands of chemical compounds and toxins. To give the best representation of the cigarette smoking phenomena, mathematicians tried to make different effective smoking models. In [5], the authors proposed for the first time a simple mathematical

\footnotetext{
*Corresponding author

Email address: jgomez@cenidet.edu.mx (J. F. Gómez-Aguilar)

doi: $10.22436 /$ jnsa.011.08.06
}

Received: 2018-01-11 Revised: 2018-03-09 Accepted: 2018-05-02 
model for giving up smoking. Sharomi and Gumel based on the model developed by Castillo-Garsow proposed a novel model introducing mild and chain classes [30]. Optimal control theory was proposed in [36], the control variables were given in the form of education campaign, anti-smoking gum, and antinicotive drugs/medicine, also were proposed for the eradication of smoking in a community. The optimal levels of the controls were characterized and the existence and uniqueness of these controls were established. In [35], the giving up smoking model taking into account the occasional smoker comportment. In [34], mathematical models were presented to study the dynamics of smoking behavior under the influence of educational programs and individual's determination to quit smoking. In [1], a smoking model that considers the number of individuals who are aware of the bad effects of smoking through education or media campaign was presented. Din [8] studied a smoking model considering non-smokers, smokers, people who temporarily or permanently quit smoking and people who are associated with illness due to smoking.

Fractional calculus (FC) is known to be a generalization of the integer-order calculus [6, 16-19, 2227, 32]. FC has attracted great attention from researchers, since fractional mathematical models are more realistic and practical than the classical integer order models. In general, the integer order model does not carry any information about the memory and learning mechanism. Otherwise, the fractional order derivatives and integrals of have nonlocal properties, so consider the past and distributed effects of any model. The nonlocal property means that the next state of a model depends not only upon its current state but also upon all its preceding states $[4,11,12,28,29,33,38]$. Therefore, any dynamical process modeled through fractional order differential equations has a memory effect. Considering the CaputoFabrizio-Caputo fractional derivative, the authors in [31] presented a new fractional giving up smoking model, the existence and uniqueness of the solution were discussed by the fixed point postulate. Zeb in [37] proposed a fractional smoking dynamic model considering adolescent nicotine dependence. In [10], the authors studied the giving up smoking dynamics using a fractional order model; approximate solutions via Laplace Adomian decomposition method were obtained. The multi-step generalized differential transform method was employed in [9] to obtain accurate solutions to a giving up smoking model of fractional order. The giving up smoking dynamics models have been extended to the scope of fractional derivatives using power law and exponential decay law.

By establishing fractional order derivative into the classical model presented in [31], we obtain the following fractional order model:

$$
\begin{aligned}
& \mathcal{D}_{t}^{\alpha} P(t)=a-a P(t)-b P(t) S(t), \\
& \mathcal{D}_{t}^{\alpha} L(t)=-a L(t)+b P(t) L(t)-c L(t) S(t), \\
& \mathcal{D}_{t}^{\alpha} S(t)=-\eta_{1} S(t)+c L(t) S(t)+f Q(t), \\
& \mathcal{D}_{t}^{\alpha} Q(t)=-\eta_{2} Q(t)+\eta_{3} S(t), \\
& \mathcal{D}_{t}^{\alpha} R(t)=-a R(t)+\eta_{4} S(t),
\end{aligned}
$$

with initial conditions

$$
\mathrm{P}(0)=\delta_{1}, \mathrm{~L}(0)=\delta_{2}, \mathrm{~S}(0)=\delta_{3}, \mathrm{Q}(0)=\delta_{4}, \mathrm{R}(0)=\delta_{5},
$$

where $\mathcal{D}_{t}^{\alpha}$ denotes the fractional derivative of the system, which in this work will be in the sense of Liouville-Caputo and Atangana-Baleanu-Caputo, $\alpha$ is a parameter that describes the order of the fractional time derivative with $0<\alpha \leqslant 1$, subject to initial conditions given in Eq. (1.2). The variables $\{P(t), L(t), S(t), Q(t), R(t)\}$ represent the potential smokers, occasional smokers, heavy smokers, temporary quitters smokers, and smokers who quit permanently. In Table 1, we present the parameters involved in the model, all parameters are positive constants reported in [31].

The modified homotopy analysis method (MHATM) was proposed in [14], this method is an analytical technique based in the combination of the homotopy analysis method and Laplace transform with homotopy polynomial. In [14], considering the Liouville-Caputo fractional derivative, the authors developed 
the MHATM method to solve time-fractional Keller-Segel model. A convergence analysis of MHATM was obtained by the proposed method and verified through different graphical representations.

Table 1: Description of parameters in system (1.1).

\begin{tabular}{|c|c|}
\hline Parameter & Description \\
\hline $\mathrm{a}$ & Rate of natural death \\
\hline $\mathrm{b}$ & Contact rate between smokers who smoke occasionally and potential smokers \\
\hline $\mathrm{c}$ & Contact rate between smokers who smoke occasionally and temporary quitters \\
\hline $\mathrm{d}$ & Rate of giving up smoking \\
\hline $\mathrm{e}$ & Remaining fraction of smokers who give up smoking forever (at a rate d) \\
\hline $\mathrm{f}$ & Contact rate between temporary quitters who return back to smoking and smokers \\
\hline$(1-e)$ & Fraction of smokers who temporarily give up smoking (at a rate $\mathrm{d})$ \\
\hline
\end{tabular}

So, we will apply the modified homotopy analysis transform method (MHATM) in Liouville-Caputo and Atangana-Baleanu-Caputo sense to compute accurate solutions of the fractional giving up smoking model. Additionally, we present special solutions of the model (1.1) which are obtained using an iterative scheme applying the Laplace transform, and the Atangana-Baleanu fractional integral. Furthermore, we analyze the uniqueness and existence of the solutions by the fixed-point theorem and Picard-Lindelof approach.

This paper is organized as follows. In Section 2, fractional order derivatives are discussed. In Section 3 , there are discussed the approximate solutions for the fractional smoking model using the ATM in Liouville-Caputo and Atangana-Baleanu-Caputo sense; also, in this section we present special solutions of the model smoking model. Uniqueness and existence of the solutions system are demonstrated by the fixed point theorem. In Section 4, numerical simulations are graphically presented. Finally, the conclusions are given in Section 5.

\section{Preliminaries}

The Liouville-Caputo fractional derivative $(\mathrm{C})$ is presented as

$$
\underset{\mathfrak{t}_{0}}{\mathrm{C}} \mathcal{D}_{\mathrm{t}}^{\alpha}\{f(t)\}=\frac{1}{\Gamma(1-\alpha)} \int_{\mathrm{t}_{0}}^{\mathrm{t}} \frac{\mathrm{d}}{\mathrm{dt}} \mathrm{f}(\tau)(\mathrm{t}-\tau)^{-\alpha} \mathrm{d} \tau, \quad \mathrm{n}-1<\alpha \leqslant \mathrm{n},
$$

where $\Gamma(\cdot)$ denotes the Gamma function.

Laplace transform to Liouville-Caputo fractional-order derivative is [27]

$$
\mathscr{L}\left\{{ }_{0}^{C} \mathcal{D}_{\mathrm{t}}^{\alpha}\{\mathrm{f}(\mathrm{t})\}\right\}(\mathrm{s})=\mathrm{S}^{\alpha} \mathrm{F}(\mathrm{S})-\sum_{\mathrm{k}=0}^{\mathrm{m}-1} \mathrm{~S}^{\alpha-k-1} \mathrm{f}^{(\mathrm{k})}(0) .
$$

Recently, Atangana and Baleanu proposed a fractional derivative with Mittag-Leffler function as the kernel of differentiation. This kernel is non-singular and nonlocal and preserves the benefits of the above Liouville-Caputo derivative [3].

The Atangana-Baleanu-Caputo fractional derivative $(\mathrm{ABC})$ is presented as

$$
{ }_{t_{0}}^{A B C} \mathcal{D}_{t}^{\alpha}\{f(t)\}=\frac{B(\alpha)}{1-\alpha} \int_{t_{0}}^{t} \frac{d}{d t} f(\tau) E_{\alpha}\left[-\alpha \frac{(t-\tau)^{\alpha}}{1-\alpha}\right] d \tau, \quad n-1<\alpha(t) \leqslant n,
$$

where $\alpha \in \mathbb{R}, \mathrm{B}(\alpha)$ denotes a normalization function $\mathrm{B}(0)=\mathrm{B}(1)=1$ and $\mathrm{E}_{\alpha}(\cdot)$ denotes the Mittag-Leffler function.

The Laplace transform of Eq. (2.2) is defined as follows

$$
\mathscr{L}\left\{\begin{array}{c}
\mathrm{ABC} \\
0
\end{array} \mathcal{D}_{\mathrm{t}}^{\alpha} \mathrm{f}(\mathrm{t})\right\}(\mathrm{s})=\frac{\mathrm{B}(\alpha)}{1-\alpha} \mathscr{L}\left[\int_{\mathrm{a}}^{\mathrm{t}} \frac{\mathrm{d}}{\mathrm{dt}} \mathrm{f}(\tau) \mathrm{E}_{\alpha}\left[-\alpha \frac{(\mathrm{t}-\tau)^{\alpha}}{1-\alpha}\right] \mathrm{d} \tau\right](\mathrm{s})=\frac{\mathrm{B}(\alpha)}{1-\alpha} \frac{\mathrm{s}^{\alpha} \mathscr{L}[\mathrm{f}(\mathrm{t})](\mathrm{s})-\mathrm{s}^{\alpha-1} \mathrm{f}(0)}{\mathrm{s}^{\alpha}+\frac{\alpha}{1-\alpha}} .
$$


The fractional integral associated to the Atangana-Baleanu derivative with non-local kernel is defined as

$$
\underset{\mathfrak{t}_{0}}{\mathrm{AB}} \mathrm{I}_{\mathfrak{t}}^{\alpha}\{f(t)\}=\frac{1-\alpha}{B(\alpha)} f(t)+\frac{\alpha}{B(\alpha) \Gamma(\alpha)} \int_{\mathrm{t}_{0}}^{\mathrm{t}} \mathrm{f}(\tau)(t-\tau)^{\alpha-1} d \tau
$$

when $\alpha$ is equal to zero the initial function is recovered and when $\alpha=1$, the classical ordinary integral is obtained.

\section{Fractional-order smoking model}

In this section, we obtain the analytical solution of the smoking model via MHATM with LiouvilleCaputo and Atangana-Baleanu fractional order derivatives in Liouville-Caputo sense. Also, we obtain special solutions using an iterative scheme via Laplace transform.

\subsection{Liouville-Caputo sense}

The modified homotopy analysis transform method (MHATM) was proposed in [14]. The method is an analytical technique based in the combination of the homotopy analysis method and Laplace transform with homotopy polynomial. The main steps of this method are described as follows:

Step 1. Let us consider the following equation

$$
\mathcal{D}_{\mathrm{t}}^{\alpha}\{f(x, t)\}+\Xi[x] f(x, t)+\Lambda[x] f(x, t)=\Psi(x, t), t>0, x \in \mathfrak{R}, \quad 0<\alpha \leqslant 1,
$$

where $\Xi[x]$ is a bounded linear operator in $x$. While the non-linear operator $\Lambda[x]$ in $x$ is Lipschitz continuous and satisfying $|\Lambda(f)-\Lambda(\phi)| \leqslant \vartheta|f-\phi|$, where $\vartheta>0$ and $\Psi(x, t)$ is a continuous function. The boundary and initial conditions can be treated in a similar way.

Step 2. Applying the methodology proposed in [13] and [15] we get the following $\mathrm{m}$-th order deformation equation

$$
\begin{aligned}
f_{m}(x, t)= & \left(x_{m}+\hbar\right) f_{m-1}-\hbar\left(1-\chi_{m}\right) \sum_{i=0}^{j-1} t^{i} f^{(i-1)}(0) \\
& +\hbar \mathcal{L}^{-1}\left(\frac{1}{s^{\alpha}} \mathcal{L}\left(\Xi_{m-1}[x] f_{m-1}(x)+\sum_{k=0}^{m-1} P_{k}\left(f_{0}, f_{1}, \ldots, f_{m}\right)-\Psi(x, t)\right)\right),
\end{aligned}
$$

where the Laplace transform is applied in Caputo sense (2.1) and $P_{k}$ is the homotopy polynomial defined by Odibat in [21].

Step 3. The non-linear term $\Lambda[x] f(x, t)$ is expanded in terms of homotopy polynomials as

$$
\Lambda[f(x, t)]=\Lambda\left(\sum_{k=0}^{m-1} f_{m}(x, t)\right)=\sum_{m=0}^{\infty} P_{m} f^{m} .
$$

Step 4. Expanding the non-linear term in (3.2) as a series of homotopy polynomials, we can calculate the various $f_{m}(x, t)$ for $m \geqslant 1$ and the solutions of Eq. (3.1) is considered as the summation of an infinite series which usually converges rapidly to the exact solutions

$$
f(x, t)=\sum_{m=0}^{\infty} f_{m}(x, t) .
$$

Following this methodology, we solved the following time-fractional smoking model via LiouvilleCaputo fractional order derivative [31]

$$
{ }_{0}^{C} \mathcal{D}_{t}^{\alpha} \mathrm{P}(\mathrm{t})=\mathrm{a}-\mathrm{aP}(\mathrm{t})-\mathrm{bP}(\mathrm{t}) \mathrm{S}(\mathrm{t}),
$$




$$
\begin{aligned}
& { }_{0}^{C} \mathcal{D}_{\mathrm{t}}^{\alpha} \mathrm{L}(\mathrm{t})=-\mathrm{aL}(\mathrm{t})+\mathrm{bP}(\mathrm{t}) \mathrm{L}(\mathrm{t})-\mathrm{cL}(\mathrm{t}) \mathrm{S}(\mathrm{t}), \\
& { }_{0}^{C} \mathcal{D}_{\mathrm{t}}^{\alpha} \mathrm{S}(\mathrm{t})=-\eta_{1} S(\mathrm{t})+\mathrm{cL}(\mathrm{t}) \mathrm{S}(\mathrm{t})+\mathrm{fQ}(\mathrm{t}), \\
& { }_{0}^{C} \mathcal{D}_{\mathrm{t}}^{\alpha} \mathrm{Q}(\mathrm{t})=-\eta_{2} \mathrm{Q}(\mathrm{t})+\eta_{3} S(\mathrm{t}), \\
& { }_{0}^{C} \mathcal{D}_{\mathrm{t}}^{\alpha} \mathrm{R}(\mathrm{t})=-\mathrm{aR}(\mathrm{t})+\eta_{4} S(\mathrm{t}),
\end{aligned}
$$

with initial conditions

$$
\mathrm{P}(0)=\delta_{1}, \quad \mathrm{~L}(0)=\delta_{2}, \quad \mathrm{~S}(0)=\delta_{3}, \quad \mathrm{Q}(0)=\delta_{4}, \quad \mathrm{R}(0)=\delta_{5},
$$

where $\eta_{1}=a-d, \eta_{2}=a+f, \eta_{3}=d(1-e), \eta_{4}=e d$.

Solution. Applying the Laplace transform (2.1) to the first equation of the system (3.3), we have

$$
s^{\alpha} \tilde{P}(s)-s^{\alpha-1} P(0)=\frac{a}{s}-\mathcal{L}\{a P(t)+b P(t) S(t)\} .
$$

Taking initial conditions and simplifying the above equation, we get

$$
\tilde{P}(s)=\frac{P(0)}{s}+\frac{a}{s^{\alpha+1}}-\frac{1}{s^{\alpha}} \mathcal{L}\{a P(t)+b P(t) S(t)\},
$$

applying the inverse Laplace transform to Eq. (3.4), we obtain

$$
\mathrm{P}(\mathrm{t})=\delta_{1}+\frac{a \mathrm{t}^{\alpha}}{\Gamma(\alpha+1)}-\mathcal{L}^{-1}\left\{\frac{1}{\mathrm{~s}^{\alpha}} \mathcal{L}\{\mathrm{aP}(\mathrm{t})+\mathrm{bP}(\mathrm{t}) \mathrm{S}(\mathrm{t})\}\right\}
$$

for the other equations shown in Eq. (3.3), we have

$$
\begin{aligned}
& L(t)=\delta_{2}-\mathcal{L}^{-1}\left\{\frac{1}{s^{\alpha}} \mathcal{L}\{a L(t)-b P(t) L(t)+c L(t) S(t)\}\right\}, \\
& S(t)=\delta_{3}-\mathcal{L}^{-1}\left\{\frac{1}{s^{\alpha}} \mathcal{L}\left\{\eta_{1} S(t)-c L(t) S(t)-f Q(t)\right\}\right\}, \\
& Q(t)=\delta_{4}-\mathcal{L}^{-1}\left\{\frac{1}{s^{\alpha}}\left\{\eta_{2} Q(t)-\eta_{3} S(t)\right\}\right\}, \\
& R(t)=\delta_{5}-\mathcal{L}^{-1}\left\{\frac{1}{s^{\alpha}}\left\{a R(t)-\eta_{4} S(t)\right\}\right\} .
\end{aligned}
$$

In this case, we choose a linear operator of the type

$$
\mathfrak{F}\left[\phi_{j}(t ; q)\right]=\mathcal{L}\left[\phi_{j}(t ; q)\right], j=1,2,
$$

with property $\mathfrak{F}(\mathfrak{c})=0$, where $c$ is constant. Next, defining the following system

$$
\begin{aligned}
& \mathrm{N}\left[\phi_{1}(\mathrm{t} ; \mathrm{q})\right]=\mathcal{L}\left[\phi_{1}(\mathrm{t} ; \mathrm{q})\right]-\delta_{1}-\frac{1}{\mathrm{~s}^{\alpha}} \mathcal{L}\left[\mathrm{a} \phi_{1}+\mathrm{b} \phi_{1} \Phi_{3}\right], \\
& \mathrm{N}\left[\Phi_{2}(\mathrm{t} ; \mathrm{q})\right]=\mathcal{L}\left[\Phi_{2}(\mathrm{t} ; \mathrm{q})\right]-\delta_{2}-\frac{1}{\mathrm{~s}^{\alpha}} \mathcal{L}\left[\mathrm{a} \Phi_{2}-\mathrm{b} \phi_{1} \Phi_{2}+\mathrm{c} \Phi_{2} \Phi_{3}\right], \\
& \mathrm{N}\left[\Phi_{3}(\mathrm{t} ; \mathrm{q})\right]=\mathcal{L}\left[\Phi_{3}(\mathrm{t} ; \mathrm{q})\right]-\delta_{3}-\frac{1}{\mathrm{~s}^{\alpha}} \mathcal{L}\left[\eta_{1} \Phi_{3}-\mathrm{c} \Phi_{2} \Phi_{3}-\mathrm{f} \Phi_{4}\right], \\
& \mathrm{N}\left[\Phi_{4}(\mathrm{t} ; \mathrm{q})\right]=\mathcal{L}\left[\Phi_{4}(\mathrm{t} ; \mathrm{q})\right]-\delta_{4}-\frac{1}{\mathrm{~s}^{\alpha}} \mathcal{L}\left[\eta_{2} \Phi_{4}-\eta_{3} \Phi_{3}\right], \\
& \mathrm{N}\left[\Phi_{5}(\mathrm{t} ; \mathrm{q})\right]=\mathcal{L}\left[\Phi_{5}(\mathrm{t} ; \mathrm{q})\right]-\delta_{5}-\frac{1}{\mathrm{~s}^{\alpha}} \mathcal{L}\left[\mathrm{a} \Phi_{5}-\eta_{4} \Phi_{3}\right] .
\end{aligned}
$$

The so-called zeroth-order deformation equation is given by

$$
(1-q) \mathfrak{F}\left[\phi_{j}(t ; q)-u_{0}(t)\right]=q \hbar N\left[\phi_{j}(t ; q)\right], \quad j=1,2,
$$


when $\mathrm{q}=0$ and $\mathrm{q}=1$, we have

$$
\phi_{j}(t ; 0)=u_{0}(t), \quad \phi_{j}(t ; 1)=u(t), \quad j=1,2,
$$

where the mth-order deformation equations are given as

$$
\begin{aligned}
\mathcal{L}\left[\mathrm{P}_{m}(t)-\chi_{m} P_{m-1}(t)\right] & =\hbar R_{m}\left(P_{m}^{\rightarrow}-1, t\right), \\
\mathcal{L}\left[L_{m}(t)-\chi_{m} L_{m-1}(t)\right] & =\hbar R_{m}\left(L_{m}^{\rightarrow}-1, t\right), \\
\mathcal{L}\left[S_{m}(t)-\chi_{m} S_{m-1}(t)\right] & =\hbar R_{m}\left(S_{m}^{\rightarrow}, 1\right), \\
\mathcal{L}\left[Q_{m}(t)-\chi_{m} Q_{m-1}(t)\right] & =\hbar R_{m}\left(Q_{m}^{\vec{m}}-1, t\right), \\
\mathcal{L}\left[R_{m}(t)-\chi_{m} R_{m-1}(t)\right] & =\hbar R_{m}\left(R_{m-1}^{\rightarrow}, t\right),
\end{aligned}
$$

applying the inverse Laplace transform to the Eq. (3.5) we have

$$
\begin{aligned}
& P_{m}(t)=\chi_{m} P_{m-1}(t)+\hbar R_{m}\left(P_{m}^{\rightarrow}-1, t\right), \\
& L_{m}(t)=\chi_{m} L_{m-1}(t)+\hbar R_{m}\left(L_{m}^{\rightarrow}-1, t\right), \\
& S_{m}(t)=\chi_{m} S_{m-1}(t)+\hbar R_{m}\left(S_{m}^{\vec{m}}-1, t\right), \\
& Q_{m}(t)=\chi_{m} Q_{m-1}(t)+\hbar R_{m}\left(Q_{m}^{\vec{m}-1}, t\right), \\
& R_{m}(t)=\chi_{m} R_{m-1}(t)+\hbar R_{m}\left(R_{m}^{\rightarrow}-1, t\right),
\end{aligned}
$$

where

$$
\begin{aligned}
& \mathrm{R}_{\mathrm{m}}\left(\mathrm{P}_{\mathrm{m}-1}^{\rightarrow}, \mathrm{t}\right)=\mathcal{L}\left[\mathrm{P}_{\mathrm{m}-1}(\mathrm{t})\right]-\left(1-\chi_{\mathrm{m}}\right)\left(\delta_{1}+\frac{a t^{\alpha}}{\Gamma(\alpha+1)}\right)-\frac{1}{\mathrm{~s}^{\alpha}} \mathcal{L}\left[\mathrm{a} \mathrm{P}_{\mathrm{m}-1}+\mathrm{b} \mathrm{H}_{\mathrm{m}}\right], \\
& \mathrm{R}_{\mathrm{m}}\left(\mathrm{L}_{\mathrm{m}-1}^{\rightarrow}, \mathrm{t}\right)=\mathcal{L}\left[\mathrm{L}_{\mathrm{m}-1}(\mathrm{t})\right]-\left(1-\chi_{\mathrm{m}}\right) \delta_{2}-\frac{1}{\mathrm{~s}^{\alpha}} \mathcal{L}\left[\mathrm{a} \mathrm{L}_{\mathrm{m}-1}-\mathrm{b} \mathrm{K}_{\mathrm{m}}^{1}+\mathrm{c} \mathrm{K}_{\mathrm{m}}^{2}\right], \\
& \mathrm{R}_{\mathrm{m}}\left(\mathrm{S}_{\mathrm{m}-1}^{\rightarrow}, \mathrm{t}\right)=\mathcal{L}\left[\mathrm{S}_{\mathrm{m}-1}(\mathrm{t})\right]-\left(1-\chi_{\mathrm{m}}\right) \delta_{3}-\frac{1}{\mathrm{~s}^{\alpha}} \mathcal{L}\left[\eta_{1} \mathrm{~S}_{\mathrm{m}-1}-\mathrm{c} \mathrm{M}_{\mathrm{m}}-\mathrm{f} \mathrm{Q}_{\mathrm{m}-1}\right], \\
& \mathrm{R}_{\mathrm{m}}\left(\mathrm{Q}_{\mathrm{m}-1}^{\rightarrow}, \mathrm{t}\right)=\mathcal{L}\left[\mathrm{Q}_{\mathrm{m}-1}(\mathrm{t})\right]-\left(1-\chi_{\mathrm{m}}\right) \delta_{4}-\frac{1}{\mathrm{~s}^{\alpha}} \mathcal{L}\left[\eta_{2} \mathrm{Q}_{\mathrm{m}-1}-\eta_{3} \mathrm{~S}_{\mathrm{m}-1}\right], \\
& \mathrm{R}_{\mathrm{m}}\left(\mathrm{R}_{\mathrm{m}-1}^{\rightarrow}, \mathrm{t}\right)=\mathcal{L}\left[\mathrm{R}_{\mathrm{m}-1}(\mathrm{t})\right]-\left(1-\chi_{\mathrm{m}}\right) \delta_{5}-\frac{1}{\mathrm{~s}^{\alpha}} \mathcal{L}\left[\mathrm{a} \mathrm{R}_{\mathrm{m}-1}-\eta_{4} S_{\mathrm{m}-1}\right] .
\end{aligned}
$$

The solution of mth-order deformation equation (3.5) is given as

$$
\begin{aligned}
& P_{m}(t)=\left(x_{m}+\hbar\right) P_{m-1}-\hbar\left(1-x_{m}\right)\left(\delta_{1}+\frac{a t^{\alpha}}{\Gamma(\alpha+1)}\right)-\hbar \mathcal{L}^{-1}\left\{\frac{1}{s^{\alpha}} \mathcal{L}\left[a P_{m-1}+b H_{m}\right]\right\}, \\
& L_{m}(t)=\left(x_{m}+\hbar\right) L_{m-1}-\hbar\left(1-x_{m}\right) \delta_{2}-\hbar \mathcal{L}^{-1}\left\{\frac{1}{s^{\alpha}} \mathcal{L}\left[a L_{m-1}-b K_{m}^{1}+c K_{m}^{2}\right]\right\}, \\
& S_{m}(t)=\left(x_{m}+\hbar\right) S_{m-1}-\hbar\left(1-x_{m}\right) \delta_{3}-\hbar \mathcal{L}^{-1}\left\{\frac{1}{s^{\alpha}} \mathcal{L}\left[\eta_{1} S_{m-1}-c M_{m}-f Q_{m-1}\right]\right\}, \\
& Q_{m}(t)=\left(x_{m}+\hbar\right) Q_{m-1}-\hbar\left(1-x_{m}\right) \delta_{4}-\hbar \mathcal{L}^{-1}\left\{\frac{1}{s^{\alpha}} \mathcal{L}\left[\eta_{2} Q_{m-1}-\eta_{3} S_{m-1}\right]\right\}, \\
& R_{m}(t)=\left(x_{m}+\hbar\right) R_{m-1}-\hbar\left(1-x_{m}\right) \delta_{5}-\hbar \mathcal{L}^{-1}\left\{\frac{1}{s^{\alpha}} \mathcal{L}\left[a R_{m-1}-\eta_{4} S_{m-1}\right]\right\},
\end{aligned}
$$

where

$$
\begin{aligned}
\mathrm{H}_{\mathrm{m}} & =\frac{1}{\Gamma(\mathrm{m}+1)}\left[\frac{\mathrm{d}^{\mathrm{m}}}{\mathrm{dq}^{\mathrm{m}}} \mathrm{N}\left[\left(\mathrm{q} \phi_{1}(\mathrm{t} ; \mathrm{q})\right)\left(\mathrm{q} \Phi_{3}(\mathrm{t} ; \mathrm{q})\right)\right]\right]_{\mathrm{q}=0^{\prime}} \\
\mathrm{K}_{\mathrm{m}}^{1} & =\frac{1}{\Gamma(\mathrm{m}+1)}\left[\frac{\mathrm{d}^{\mathrm{m}}}{\mathrm{dq}^{\mathrm{m}}} \mathrm{N}\left[\left(\mathrm{q} \phi_{1}(\mathrm{t} ; \mathrm{q})\right)\left(\mathrm{q} \Phi_{2}(\mathrm{t} ; \mathrm{q})\right)\right]\right]_{\mathrm{q}=0^{\prime}} \\
\mathrm{K}_{\mathrm{m}}^{2} & =\frac{1}{\Gamma(\mathrm{m}+1)}\left[\frac{\mathrm{d}^{\mathrm{m}}}{\mathrm{dq}^{\mathrm{m}}} \mathrm{N}\left[\left(\mathrm{q} \Phi_{2}(\mathrm{t} ; \mathrm{q})\right)\left(\mathrm{q} \Phi_{3}(\mathrm{t} ; \mathrm{q})\right)\right]\right]_{\mathrm{q}=0^{\prime}} \\
M_{\mathrm{m}} & =\frac{1}{\Gamma(\mathrm{m}+1)}\left[\frac{\mathrm{d}^{\mathrm{m}}}{\mathrm{dq}^{\mathrm{m}}} \mathrm{N}\left[\left(\mathrm{q} \Phi_{2}(\mathrm{t} ; \mathrm{q})\right)\left(\mathrm{q} \Phi_{3}(\mathrm{t} ; \mathrm{q})\right)\right]\right]_{\mathrm{q}=0}
\end{aligned}
$$


Taking the initial conditions and the iterative scheme (3.6), we obtain the following iterations

$$
\begin{aligned}
& p^{0}: P_{0}(t)=\delta_{1}+\frac{a t^{\alpha}}{\Gamma(\alpha+1)}, \quad L_{0}(t)=\delta_{2}, \quad S_{0}(t)=\delta_{3}, \quad Q_{0}(t)=\delta_{4}, \quad R_{0}(t)=\delta_{5}, \\
& p^{1}: P_{1}(x, t)=m_{1} \frac{\hbar t^{2 \alpha}}{\Gamma(2 \alpha+1)}+m_{2} \frac{\hbar t^{\alpha}}{\Gamma(\alpha+1)}, \\
& \mathrm{L}_{1}(\mathrm{t})=\mathrm{m}_{3} \frac{\hbar \mathrm{t}^{\alpha}}{\Gamma(\alpha+1)}-\mathrm{m}_{4} \frac{\hbar \mathrm{t}^{2 \alpha}}{\Gamma(2 \alpha+1)}, \\
& S_{1}(t)=m_{5} \frac{\hbar t^{\alpha}}{\Gamma(\alpha+1)} \\
& \mathrm{Q}_{1}(\mathrm{t})=\mathrm{m}_{6} \frac{\hbar \mathrm{t}^{\alpha}}{\Gamma(\alpha+1)} \\
& \mathrm{R}_{1}(\mathrm{t})=\mathrm{m}_{7} \frac{\hbar \mathrm{t}^{\alpha}}{\Gamma(\alpha+1)} \\
& p^{2}: P_{2}(t)=m_{1} \frac{\hbar(1+\hbar) t^{2 \alpha}}{\Gamma(2 \alpha+1)}+m_{2} \frac{\hbar(1+\hbar) t^{\alpha}}{\Gamma(\alpha+1)}+\left(a m_{2}+b\left(\delta_{1} m_{5}+\delta_{3} m_{2}\right)\right) \frac{\hbar^{2} t^{2 \alpha}}{\Gamma(2 \alpha+1)} \\
& +\left(a m_{1}+b\left(\frac{a m_{5}}{\Gamma(\alpha+1)}+\delta_{3} m_{1}\right)\right) \frac{\hbar^{2} t^{3 \alpha}}{\Gamma(3 \alpha+1)} \\
& \mathrm{L}_{2}(\mathrm{t})=\mathrm{m}_{3} \frac{\hbar(1+\hbar) \mathrm{t}^{\alpha}}{\Gamma(\alpha+1)}-\mathrm{m}_{4} \frac{\hbar(1+\hbar) \mathrm{t}^{2 \alpha}}{\Gamma(2 \alpha+1)} \\
& +\left(a m_{3}-b\left(\delta_{1} m_{3}+\delta_{2} m_{2}\right)+c\left(\delta_{3} m_{3}+\delta_{2} m_{5}\right)\right) \frac{\hbar^{2} t^{2 \alpha}}{\Gamma(2 \alpha+1)} \\
& +\left(a m_{4}-\left(\delta_{1} m_{4}-a m_{3}-\delta_{2} m_{1}\right)+c \delta_{3} m_{4}\right) \frac{\hbar^{2} t^{3 \alpha}}{\Gamma(3 \alpha+1)} \\
& -\frac{\operatorname{abm}_{4} \Gamma(3 \alpha+1)}{\Gamma(\alpha+1) \Gamma(2 \alpha+1)} \frac{\hbar^{2} t^{4 \alpha}}{\Gamma(4 \alpha+1)} \\
& S_{2}(t)=m_{5} \frac{\hbar(1+\hbar) t^{\alpha}}{\Gamma(\alpha+1)}+\left(m_{5} \eta_{1}-c\left(\delta_{3} m_{3}+\delta_{2} m_{5}\right)-f m_{6}\right) \frac{\hbar^{2} t^{2 \alpha}}{\Gamma(2 \alpha+1)}+c \delta_{3} m_{4} \frac{\hbar^{2} t^{3 \alpha}}{\Gamma(3 \alpha+1)} \text {, } \\
& \mathrm{Q}_{2}(\mathrm{t})=\mathrm{m}_{6} \frac{\hbar(1+\hbar) \mathrm{t}^{\alpha}}{\Gamma(\alpha+1)}+\left(\eta_{2} \mathrm{~m}_{6}-\eta_{3} \mathrm{~m}_{5}\right) \frac{\hbar^{2} \mathrm{t}^{2 \alpha}}{\Gamma(2 \alpha+1)}, \\
& R_{2}(t)=m_{7} \frac{\hbar(1+\hbar) t^{\alpha}}{\Gamma(\alpha+1)}+\left(a m_{7}-\eta_{4} m_{5}\right) \frac{\hbar^{2} t^{2 \alpha}}{\Gamma(2 \alpha+1)},
\end{aligned}
$$

where $m_{j}, j=1,2 \ldots, 7$, are given by

$$
\begin{array}{lll}
m_{1}=a^{2}+a b \delta_{3}, & m_{2}=a \delta_{1}+b \delta_{1} \delta_{3}, & m_{3}=\delta_{2}\left(a-b \delta_{1}+c \delta_{3}\right), \\
m_{4}=a b \delta_{2}, & m_{5}=\left(\eta_{1} \delta_{3}-c \delta_{2} \delta_{3}-f \delta_{4}\right), & m_{6}=\left(\eta_{2} \delta_{4}-\delta_{3} \delta_{3}\right), \\
m_{7}=\left(a \delta_{5}-\delta_{4} \delta_{3}\right) . & &
\end{array}
$$

Finally, the solutions of the Eq. (3.3) are given by

$$
\begin{aligned}
& \mathrm{P}(\mathrm{t})=\mathrm{P}_{0}(\mathrm{t})+\mathrm{P}_{1}(\mathrm{t})+\mathrm{P}_{2}(\mathrm{t})+\cdots=\sum_{\mathrm{m}=0}^{\infty} \mathrm{P}_{\mathrm{m}}(\mathrm{t}), \\
& \mathrm{L}(\mathrm{t})=\mathrm{L}_{0}(\mathrm{t})+\mathrm{L}_{1}(\mathrm{t})+\mathrm{L}_{2}(\mathrm{t})+\cdots=\sum_{\mathrm{m}=0}^{\infty} \mathrm{L}_{\mathrm{m}}(\mathrm{t}),
\end{aligned}
$$




$$
\begin{aligned}
& S(t)=S_{0}(t)+S_{1}(t)+S_{2}(t)+\cdots=\sum_{m=0}^{\infty} S_{m}(t), \\
& Q(t)=Q_{0}(t)+Q_{1}(t)+Q_{2}(t)+\cdots=\sum_{m=0}^{\infty} Q_{m}(t), \\
& R(t)=R_{0}(t)+R_{1}(t)+R_{2}(t)+\cdots=\sum_{m=0}^{\infty} R_{m}(t) .
\end{aligned}
$$

Another solution of the model (3.3) can be obtained coupling the Laplace transform (2.1) and its inverse. The iterative scheme is given by

$$
\begin{aligned}
& \mathrm{P}_{(\mathrm{n})}(\mathrm{t})=\delta_{1}+\mathscr{L}^{-1}\left\{\frac{1}{\mathrm{~s}^{\gamma}} \mathscr{L}\left[\mathrm{a}\left(1-\mathrm{P}_{(\mathrm{n}-1)}(\mathrm{t})\right)-\mathrm{b} \mathrm{P}_{(\mathrm{n}-1)}(\mathrm{t}) \mathrm{S}_{(\mathrm{n}-1)}(\mathrm{t})\right](\mathrm{s})\right\}(\mathrm{t}), \\
& \mathrm{L}_{(\mathrm{n})}(\mathrm{t})=\delta_{2}+\mathscr{L}^{-1}\left\{\frac{1}{\mathrm{~s}^{\gamma}} \mathscr{L}\left[-\mathrm{a} \mathrm{L}_{(\mathrm{n}-1)}(\mathrm{t})+\mathrm{b} \mathrm{P}_{(\mathrm{n}-1)}(\mathrm{t}) \mathrm{L}_{(\mathrm{n}-1)}(\mathrm{t})-\mathrm{cL}(\mathrm{n}-1)(\mathrm{t}) \mathrm{S}_{(\mathrm{n}-1)}(\mathrm{t})\right](\mathrm{s})\right\}(\mathrm{t}), \\
& \mathrm{S}_{(\mathrm{n})}(\mathrm{t})=\delta_{3}+\mathscr{L}^{-1}\left\{\frac{1}{\mathrm{~s}^{\gamma}} \mathscr{L}\left[-\eta_{1} \mathrm{~S}_{(\mathrm{n}-1)}(\mathrm{t})+\mathrm{cL} \mathrm{L}_{(\mathrm{n}-1)}(\mathrm{t}) \mathrm{S}_{(\mathrm{n}-1)}(\mathrm{t})+\mathrm{fQ}(\mathrm{n}-1)(\mathrm{t})\right](\mathrm{s})\right\}(\mathrm{t}), \\
& \mathrm{Q}_{(\mathrm{n})}(\mathrm{t})=\delta_{4}+\mathscr{L}^{-1}\left\{\frac{1}{\mathrm{~s}^{\gamma}} \mathscr{L}\left[-\eta_{2} \mathrm{Q}_{(\mathrm{n}-1)}(\mathrm{t})+\eta_{3} \mathrm{~S}_{(\mathrm{n}-1)}(\mathrm{t})\right](\mathrm{s})\right\}(\mathrm{t}), \\
& \mathrm{R}_{1(\mathrm{n})}(\mathrm{t})=\delta_{5}+\mathscr{L}^{-1}\left\{\frac{1}{\mathrm{~s}^{\gamma}} \mathscr{L}\left[-\mathrm{aR}_{(\mathrm{n}-1)}(\mathrm{t})+\eta_{4} \mathrm{~S}_{(\mathrm{n}-1)}(\mathrm{t})\right](\mathrm{s})\right\}(\mathrm{t}),
\end{aligned}
$$

where $\delta_{1}, \delta_{2}, \delta_{3}, \delta_{4}$ and $\delta_{1}$ are the initial conditions.

The solution is assumed to be obtain as a limit when $n$ tends to infinity

$$
\begin{array}{ll}
\mathrm{P}(\mathrm{t})=\lim _{n \rightarrow \infty} \mathrm{P}_{(\mathrm{n})}(\mathrm{t}) ; & \mathrm{L}(\mathrm{t})=\lim _{n \rightarrow \infty} \mathrm{L}_{(\mathrm{n})}(\mathrm{t}) ; \quad \mathrm{S}(\mathrm{t})=\lim _{\mathrm{n} \rightarrow \infty} \mathrm{S}_{(\mathrm{n})}(\mathrm{t}) ; \\
\mathrm{Q}(\mathrm{t})=\lim _{\mathrm{n} \rightarrow \infty} \mathrm{Q}_{(\mathrm{n})}(\mathrm{t}) ; & \mathrm{R}_{1}(\mathrm{t})=\lim _{\mathrm{n} \rightarrow \infty} \mathrm{R}_{1(\mathrm{n})}(\mathrm{t}) .
\end{array}
$$

Theorem 3.1. The recursive method given by Eq. (3.8) is stable.

Proof. We assume the following. It is possible to find five positive constants $A, B, C, D$, and $E$ such that for all $0 \leqslant t \leqslant T \leqslant \infty$,

$$
\|\mathrm{P}(\mathrm{t})\|<\mathrm{A} ; \quad\|\mathrm{L}(\mathrm{t})\|<\mathrm{B} ; \quad\|\mathrm{S}(\mathrm{t})\|<\mathrm{C} ; \quad\|\mathrm{Q}(\mathrm{t})\|<\mathrm{D} ; \quad \text { and } \quad\|\mathrm{R}(\mathrm{t})\|<\mathrm{E} .
$$

Now, we consider a subset of $\mathrm{L}_{2}((\mathrm{a}, \mathrm{b})(0, \mathrm{~T}))$ defined by

$$
\Xi=\left\{\eta:(a, b)(0, T) \rightarrow \Xi, \quad \frac{1}{\Gamma(\alpha)} \int(t-\eta)^{\alpha-1} v(\eta) u(\eta) d \eta<\infty\right\},
$$

considering the following operator $\Theta$ is defined as

$$
\Theta(P, L, S, Q, R)=\left\{\begin{array}{l}
a(1-P(t))-b P(t) S(t), \\
-a L(t)+b P(t) L(t)-c L(t) S(t), \\
-\eta_{1} S(t)+c L(t) S(t)+f Q(t), \\
-\eta_{2} Q(t)+\eta_{3} S(t), \\
-a R(t)+\eta_{4} S(t) .
\end{array}\right.
$$

Then

$$
=\left\{\begin{array}{l}
<\Theta(P, L, S, Q, R)-\Theta\left(P_{1}, L_{1}, S_{1}, Q_{1}, R_{1}\right),\left(P-P_{1}, L-L_{1}, S-S_{1}, Q-Q_{1}, R-R_{1}\right)> \\
<a\left(1-\left(P(t)-P_{1}(t)\right)\right)-b\left(P(t)-P_{1}(t)\right)\left(S(t)-S_{1}(t)\right),\left(P(t)-P_{1}(t)\right)> \\
<-a\left(L(t)-L_{1}(t)\right)+b\left(P(t)-P_{1}(t)\right)\left(L(t)-L_{1}(t)\right)-c\left(L(t)-L_{1}(t)\right)\left(S(t)-S_{1}(t)\right),\left(L(t)-L_{1}(t)\right)> \\
<-\eta_{1}\left(S(t)-S_{1}(t)\right)+c\left(L(t)-L_{1}(t)\right)\left(S(t)-S_{1}(t)\right)+f\left(Q(t)-Q_{1}(t)\right),\left(S(t)-S_{1}(t)\right)> \\
<-\eta_{2}\left(Q(t)-Q_{1}(t)\right)+\eta_{3}\left(S(t)-S_{1}(t)\right),\left(Q(t)-Q_{1}(t)\right)>, \\
<-a\left(R(t)-R_{1}(t)\right)+\eta_{4}\left(S(t)-S_{1}(t)\right)>
\end{array}\right.
$$


where,

$$
\mathrm{P}(\mathrm{t}) \neq \mathrm{P}_{1}(\mathrm{t}) ; \quad \mathrm{L}(\mathrm{t}) \neq \mathrm{L}_{1}(\mathrm{t}) ; \quad \mathrm{S}(\mathrm{t}) \neq \mathrm{S}_{1}(\mathrm{t}) ; \quad \mathrm{Q}(\mathrm{t}) \neq \mathrm{Q}_{1}(\mathrm{t}) ; \quad \mathrm{R}(\mathrm{t}) \neq \mathrm{R}_{1}(\mathrm{t}) .
$$

Thus, applying the norm and the absolute value in both sides, we have

$$
\begin{aligned}
& <\Theta(P, L, S, Q, R)-\Theta\left(P_{1}, L_{1}, S_{1}, Q_{1}, R_{1}\right),\left(P-P_{1}, L-L_{1}, S-S_{1}, Q-Q_{1}, R-R_{1}\right)>,
\end{aligned}
$$

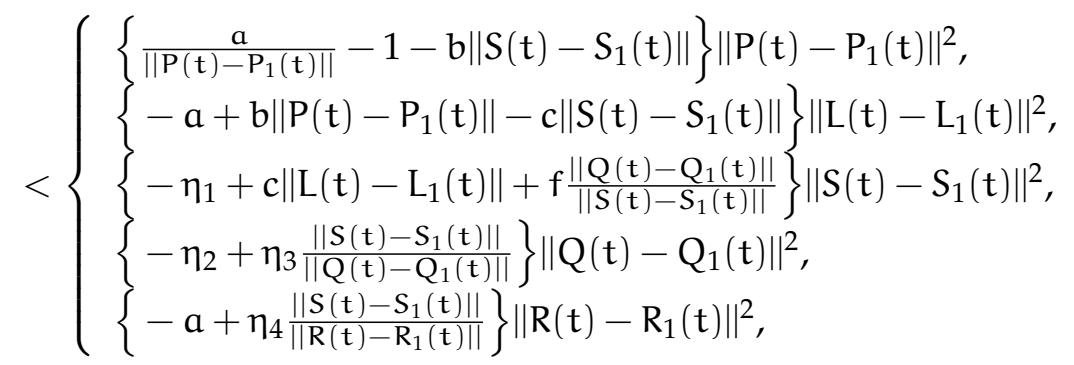

where

$$
\begin{aligned}
& <\Theta(P, L, S, Q, R)-\Theta\left(P_{1}, L_{1}, S_{1}, Q_{1}, R_{1}\right),\left(P-P_{1}, L-L_{1}, S-S_{1}, Q-Q_{1}, R-R_{1}\right)>, \\
& \quad<\left\{\begin{array}{l}
M\left\|P(t)-P_{1}(t)\right\|^{2} \\
N\left\|L(t)-L_{1}(t)\right\|^{2} \\
O\left\|S(t)-S_{1}(t)\right\|^{2} \\
T\left\|Q(t)-Q_{1}(t)\right\|^{2} \\
V\left\|R(t)-R_{1}(t)\right\|^{2}
\end{array}\right.
\end{aligned}
$$

with

$$
\begin{aligned}
& \mathrm{M}=\frac{\mathrm{a}}{\left\|\mathrm{P}(\mathrm{t})-\mathrm{P}_{1}(\mathrm{t})\right\|}-1-\mathrm{b}\left\|\mathrm{S}(\mathrm{t})-\mathrm{S}_{1}(\mathrm{t})\right\|, \\
& \mathrm{N}=-\mathrm{a}+\mathrm{b}\left\|\mathrm{P}(\mathrm{t})-\mathrm{P}_{1}(\mathrm{t})\right\|-\mathrm{c}\left\|\mathrm{S}(\mathrm{t})-\mathrm{S}_{1}(\mathrm{t})\right\|, \\
& \mathrm{O}=-\eta_{1}+\mathrm{c}\left\|\mathrm{L}(\mathrm{t})-\mathrm{L}_{1}(\mathrm{t})\right\|+\mathrm{f} \frac{\left\|\mathrm{Q}(\mathrm{t})-\mathrm{Q}_{1}(\mathrm{t})\right\|}{\left\|\mathrm{S}(\mathrm{t})-\mathrm{S}_{1}(\mathrm{t})\right\|}, \\
& \mathrm{T}=-\eta_{2}+\eta_{3} \frac{\left\|\mathrm{S}(\mathrm{t})-\mathrm{S}_{1}(\mathrm{t})\right\|}{\left\|\mathrm{Q}(\mathrm{t})-\mathrm{Q}_{1}(\mathrm{t})\right\|}, \\
& \mathrm{Q}=-\mathrm{a}+\eta_{4} \frac{\left\|\mathrm{S}(\mathrm{t})-\mathrm{S}_{1}(\mathrm{t})\right\|}{\left\|\mathrm{R}(\mathrm{t})-\mathrm{R}_{1}(\mathrm{t})\right\|}
\end{aligned}
$$

Also if we consider a given non-null vector $\left(P_{1}, L_{1}, S_{1}, Q_{1}, R_{1}\right)$, using some routine as above case, we obtain

$$
\begin{aligned}
& <\Theta(P, L, S, Q, R)-\Omega\left(P_{1}, L_{1}, S_{1}, Q_{1}, R_{1}\right),\left(P-P_{1}, L-L_{1}, S-S_{1}, Q-Q_{1}, R-R_{1}\right)>, \\
& \quad<\left\{\begin{array}{l}
M\left\|P(t)-P_{1}(t)\right\|\|\mid P(t)\|, \\
N\left\|L(t)-L_{1}(t)\right\|\|L(t)\|, \\
O\left\|S(t)-S_{1}(t)\right\|\|S(t)\|, \\
T\left\|Q(t)-Q_{1}(t)\right\|\|Q(t)\|, \\
V\left\|R(t)-R_{1}(t)\right\|\|R(t)\|,
\end{array}\right.
\end{aligned}
$$

from the results obtained in Eqs. (3.9) and (3.10), we conclude that the used iterative method is stable.

Now we can propose a solution for system given by Eq. (3.3) using the predictor-corrector AdamsBashforth-Moulton method as follows [20] and [7]

$$
\begin{aligned}
& P(t)=\sum_{u=0}^{n-1} \delta_{1}^{u} \frac{t^{u}}{u !}+\frac{1}{\Gamma(\alpha)} \int_{0}^{t}(t-\tau)^{\alpha-1}[a(1-P(\tau))-b P(\tau) S(\tau)] d u, \\
& L(t)=\sum_{u=0}^{n-1} \delta_{2}^{u} \frac{t^{u}}{u !}+\frac{1}{\Gamma(\alpha)} \int_{0}^{t}(t-\tau)^{\alpha-1}[-a L(\tau)+b P(\tau) L(\tau)-c L(\tau) S(\tau)] d u,
\end{aligned}
$$




$$
\begin{aligned}
& S(t)=\sum_{u=0}^{n-1} \delta_{3}^{u} \frac{t^{u}}{u !}+\frac{1}{\Gamma(\alpha)} \int_{0}^{t}(t-\tau)^{\alpha-1}\left[-\eta_{1} S(\tau)+c L(\tau) S(\tau)+f Q(\tau)\right] d u, \\
& Q(t)=\sum_{u=0}^{n-1} \delta_{4}^{u} \frac{t^{u}}{u !}+\frac{1}{\Gamma(\alpha)} \int_{0}^{t}(t-\tau)^{\alpha-1}\left[-\eta_{2} Q(\tau)+\eta_{3} S(\tau)\right] d u, \\
& R(t)=\sum_{u=0}^{n-1} \delta_{5}^{u} \frac{t^{u}}{u !}+\frac{1}{\Gamma(\alpha)} \int_{0}^{t}(t-\tau)^{\alpha-1}\left[-a R(\tau)+\eta_{4} S(\tau)\right] d u .
\end{aligned}
$$

\subsection{Atangana-Baleanu-Caputo sense}

Following the methodology described in [14] we solve the following time-fractional smoking model via Atangana-Baleanu-Caputo fractional order derivative

$$
\begin{aligned}
& { }_{0}^{A B C} \mathcal{D}_{t}^{\alpha} P(t)=a-a P(t)-b P(t) S(t), \\
& { }_{0}^{A B C} \mathcal{D}_{t}^{\alpha} L(t)=-a L(t)+b P(t) L(t)-c L(t) S(t), \\
& { }_{0}^{A B C} \mathcal{D}_{t}^{\alpha} S(t)=-\eta_{1} S(t)+c L(t) S(t)+f Q(t), \\
& { }_{0}^{A B C} \mathcal{D}_{t}^{\alpha} Q(t)=-\eta_{2} Q(t)+\eta_{3} S(t), \\
& { }_{0}^{A B C} \mathcal{D}_{t}^{\alpha} R(t)=-a R(t)+\eta_{4} S(t),
\end{aligned}
$$

with initial conditions

$$
\mathrm{P}(0)=\delta_{1}, \quad \mathrm{~L}(0)=\delta_{2}, \quad \mathrm{~S}(0)=\delta_{3}, \quad \mathrm{Q}(0)=\delta_{4}, \quad \mathrm{R}(0)=\delta_{5},
$$

where $\eta_{1}=a-d, \eta_{2}=a+f, \eta_{3}=d(1-e), \eta_{4}=e d$.

solution. Applying the Laplace transform (2.3) to the first equation of the system (3.12), we have

$$
\frac{B(\alpha)}{1-\alpha} \frac{s^{\alpha} \tilde{P}(s)-s^{\alpha-1} P(0)}{s^{\alpha}+\frac{\alpha}{1-\alpha}}=\frac{a}{s}-\mathcal{L}\{a P(t)+b P(t) S(t)\}
$$

Taking initial conditions and simplifying the above equation, we get

$$
\tilde{P}(s)=\frac{P(0)}{s}+\frac{a(1-\alpha)}{B(\alpha) s}+\frac{a \alpha}{B(\alpha) s^{\alpha+1}}-\frac{(1-\alpha) s^{\alpha}+\alpha}{B(\alpha) s^{\alpha}} \mathcal{L}\{a P(t)+b P(t) S(t)\},
$$

applying the inverse Laplace transform to Eq. (3.13), we obtain

$$
\mathrm{P}(\mathrm{t})=\delta_{1}+\frac{\mathrm{a}(1-\alpha)}{\mathrm{B}(\alpha)}+\frac{\mathrm{a} \alpha \mathrm{t}^{\alpha}}{\mathrm{B}(\alpha) \Gamma(\alpha+1)}-\mathcal{L}^{-1}\left\{\frac{(1-\alpha) \mathrm{s}^{\alpha}+\alpha}{\mathrm{B}(\alpha) \mathrm{s}^{\alpha}} \mathcal{L}\{\mathrm{aP}(\mathrm{t})+\mathrm{bP}(\mathrm{t}) \mathrm{S}(\mathrm{t})\}\right\},
$$

for the other equations shown in (3.12), we have

$$
\begin{aligned}
& L(t)=\delta_{2}-\mathcal{L}^{-1}\left\{\frac{(1-\alpha) s^{\alpha}+\alpha}{B(\alpha) s^{\alpha}} \mathcal{L}\{a L(t)-b P(t) L(t)+c L(t) S(t)\}\right\}, \\
& S(t)=\delta_{3}-\mathcal{L}^{-1}\left\{\frac{(1-\alpha) s^{\alpha}+\alpha}{B(\alpha) s^{\alpha}} \mathcal{L}\left\{\eta_{1} S(t)-c L(t) S(t)-f Q(t)\right\}\right\}, \\
& Q(t)=\delta_{4}-\mathcal{L}^{-1}\left\{\frac{(1-\alpha) s^{\alpha}+\alpha}{B(\alpha) s^{\alpha}}\left\{\eta_{2} Q(t)-\eta_{3} S(t)\right\}\right\}, \\
& R(t)=\delta_{5}-\mathcal{L}^{-1}\left\{\frac{(1-\alpha) s^{\alpha}+\alpha}{B(\alpha) s^{\alpha}}\left\{a R(t)-\eta_{4} S(t)\right\}\right\} .
\end{aligned}
$$


In this case, we choose a linear operator of the type

$$
\mathfrak{F}\left[\phi_{j}(t ; q)\right]=\mathcal{L}\left[\phi_{j}(t ; q)\right], j=1,2
$$

with property $\mathfrak{F}(\mathrm{c})=0$, where $\mathrm{c}$ is constant. Next, we define the following system

$$
\begin{aligned}
& \mathrm{N}\left[\phi_{1}(\mathrm{t} ; \mathrm{q})\right]=\mathcal{L}\left[\phi_{1}(\mathrm{t} ; \mathrm{q})\right]-\delta_{1}-\frac{(1-\alpha) \mathrm{s}^{\alpha}+\alpha}{\mathrm{B}(\alpha) \mathrm{s}^{\alpha}} \mathcal{L}\left[\mathrm{a} \phi_{1}+\mathrm{b} \phi_{1} \Phi_{3}\right], \\
& \mathrm{N}\left[\Phi_{2}(\mathrm{t} ; \mathrm{q})\right]=\mathcal{L}\left[\Phi_{2}(\mathrm{t} ; \mathrm{q})\right]-\delta_{2}-\frac{(1-\alpha) s^{\alpha}+\alpha}{\mathrm{B}(\alpha) s^{\alpha}} \mathcal{L}\left[\mathrm{a} \Phi_{2}-\mathrm{b} \phi_{1} \Phi_{2}+\mathrm{c} \Phi_{2} \Phi_{3}\right] \\
& \mathrm{N}\left[\Phi_{3}(\mathrm{t} ; \mathrm{q})\right]=\mathcal{L}\left[\Phi_{3}(\mathrm{t} ; \mathrm{q})\right]-\delta_{3}-\frac{(1-\alpha) s^{\alpha}+\alpha}{\mathrm{B}(\alpha) s^{\alpha}} \mathcal{L}\left[\eta_{1} \Phi_{3}-\mathrm{c} \Phi_{2} \Phi_{3}-\mathrm{f} \Phi_{4}\right], \\
& \mathrm{N}\left[\Phi_{4}(\mathrm{t} ; \mathrm{q})\right]=\mathcal{L}\left[\Phi_{4}(\mathrm{t} ; \mathrm{q})\right]-\delta_{4}-\frac{(1-\alpha) s^{\alpha}+\alpha}{\mathrm{B}(\alpha) s^{\alpha}} \mathcal{L}\left[\eta_{2} \Phi_{4}-\eta_{3} \Phi_{3}\right] \\
& \mathrm{N}\left[\Phi_{5}(\mathrm{t} ; \mathrm{q})\right]=\mathcal{L}\left[\Phi_{5}(\mathrm{t} ; \mathrm{q})\right]-\delta_{5}-\frac{(1-\alpha) s^{\alpha}+\alpha}{\mathrm{B}(\alpha) s^{\alpha}} \mathcal{L}\left[\mathrm{a} \Phi_{5}-\eta_{4} \Phi_{3}\right] .
\end{aligned}
$$

The so-called zeroth-order deformation equation is given by

$$
(1-q) \mathfrak{F}\left[\phi_{j}(t ; q)-u_{0}(t)\right]=q \hbar N\left[\phi_{j}(t ; q)\right], \quad j=1,2,
$$

when $\mathrm{q}=0$ and $\mathrm{q}=1$, we have

$$
\phi_{j}(t ; 0)=u_{0}(t), \quad \phi_{j}(t ; 1)=u(t), j=1,2,
$$

where the mth-order deformation equations are given as

$$
\begin{aligned}
\mathcal{L}\left[\mathrm{P}_{m}(t)-\chi_{m} P_{m-1}(t)\right] & =\hbar R_{m}\left(P_{m}^{\rightarrow}-1, t\right), \\
\mathcal{L}\left[L_{m}(t)-\chi_{m} L_{m-1}(t)\right] & =\hbar R_{m}\left(L_{m}^{\rightarrow}-1, t\right), \\
\mathcal{L}\left[S_{m}(t)-\chi_{m} S_{m-1}(t)\right] & =\hbar R_{m}\left(S_{m}^{\rightarrow}, 1\right), \\
\mathcal{L}\left[Q_{m}(t)-\chi_{m} Q_{m-1}(t)\right] & =\hbar R_{m}\left(Q_{m}^{\vec{m}}-1, t\right), \\
\mathcal{L}\left[R_{m}(t)-\chi_{m} R_{m-1}(t)\right] & =\hbar R_{m}\left(R_{m-1}^{\rightarrow}, t\right),
\end{aligned}
$$

applying the inverse Laplace transform to Eq. (3.14) we get

$$
\begin{aligned}
P_{m}(t) & =\chi_{m} P_{m-1}(t)+\hbar R_{m}\left(P_{m}^{\rightarrow}-1, t\right), \\
L_{m}(t) & =\chi_{m} L_{m-1}(t)+\hbar R_{m}\left(L_{m}-1, t\right), \\
S_{m}(t) & =\chi_{m} S_{m-1}(t)+\hbar R_{m}\left(S_{m}^{\rightarrow}-1, t\right), \\
Q_{m}(t) & =\chi_{m} Q_{m-1}(t)+\hbar R_{m}\left(Q_{m}^{\vec{m}}-1, t\right), \\
R_{m}(t) & =\chi_{m} R_{m-1}(t)+\hbar R_{m}\left(R_{m}^{\rightarrow}-1, t\right),
\end{aligned}
$$

where

$$
\begin{aligned}
& \mathrm{R}_{\mathrm{m}}\left(\mathrm{P}_{\mathrm{m}-1}^{\rightarrow}, \mathrm{t}\right)=\mathcal{L}\left[\mathrm{P}_{\mathrm{m}-1}(\mathrm{t})\right]-\left(1-\chi_{\mathrm{m}}\right)\left(\delta_{1}+\frac{\mathrm{a}(1-\alpha)}{\mathrm{B}(\alpha)}+\frac{\mathrm{a} \alpha \mathrm{t}^{\alpha}}{\mathrm{B}(\alpha) \Gamma(\alpha+1)}\right) \\
& -\frac{(1-\alpha) s^{\alpha}+\alpha}{B(\alpha) s^{\alpha}} \mathcal{L}\left[a P_{m-1}+b H_{m}\right] \\
& R_{m}\left(L_{m}-1, t\right)=\mathcal{L}\left[L_{m-1}(t)\right]-\left(1-\chi_{m}\right) \delta_{2}-\frac{(1-\alpha) s^{\alpha}+\alpha}{B(\alpha) s^{\alpha}} \mathcal{L}\left[a L_{m-1}-b K_{m}^{1}+c K_{m}^{2}\right], \\
& R_{m}\left(S_{m-1}^{\rightarrow}, t\right)=\mathcal{L}\left[S_{m-1}(t)\right]-\left(1-\chi_{m}\right) \delta_{3}-\frac{(1-\alpha) s^{\alpha}+\alpha}{B(\alpha) s^{\alpha}} \mathcal{L}\left[\eta_{1} S_{m-1}-c M_{m}-f Q_{m-1}\right],
\end{aligned}
$$




$$
\begin{aligned}
& R_{m}\left(Q_{m-1}^{\rightarrow}, t\right)=\mathcal{L}\left[Q_{m-1}(t)\right]-\left(1-\chi_{m}\right) \delta_{4}-\frac{(1-\alpha) s^{\alpha}+\alpha}{B(\alpha) s^{\alpha}} \mathcal{L}\left[\eta_{2} Q_{m-1}-\eta_{3} S_{m-1}\right], \\
& R_{m}\left(R_{m-1}^{\rightarrow}, t\right)=\mathcal{L}\left[R_{m-1}(t)\right]-\left(1-\chi_{m}\right) \delta_{5}-\frac{(1-\alpha) s^{\alpha}+\alpha}{B(\alpha) s^{\alpha}} \mathcal{L}\left[a R_{m-1}-\eta_{4} s_{m-1}\right] .
\end{aligned}
$$

The solution of mth-order deformation equations (3.14) are given as

$$
\begin{aligned}
P_{m}(t)= & \left(\chi_{m}+\hbar\right) P_{m-1}-\hbar\left(1-\chi_{m}\right)\left(\delta_{1}+\frac{a(1-\alpha)}{B(\alpha)}+\frac{a \alpha t^{\alpha}}{B(\alpha) \Gamma(\alpha+1)}\right) \\
& -\hbar \mathcal{L}^{-1}\left\{\frac{(1-\alpha) s^{\alpha}+\alpha}{B(\alpha) s^{\alpha}} \mathcal{L}\left[a P_{m-1}+b H_{m}\right]\right\}, \\
L_{m}(t)= & \left(\chi_{m}+\hbar\right) L_{m-1}-\hbar\left(1-\chi_{m}\right) \delta_{2}-\hbar \mathcal{L}^{-1}\left\{\frac{(1-\alpha) s^{\alpha}+\alpha}{B(\alpha) s^{\alpha}} \mathcal{L}\left[a L_{m-1}-b K_{m}^{1}+c K_{m}^{2}\right]\right\}, \\
S_{m}(t)= & \left(\chi_{m}+\hbar\right) S_{m-1}-\hbar\left(1-\chi_{m}\right) \delta_{3}-\hbar \mathcal{L}^{-1}\left\{\frac{(1-\alpha) s^{\alpha}+\alpha}{B(\alpha) s^{\alpha}} \mathcal{L}\left[\eta_{1} S_{m-1}-c M_{m}-f Q_{m-1}\right]\right\}, \\
Q_{m}(t)= & \left(\chi_{m}+\hbar\right) Q_{m-1}-\hbar\left(1-\chi_{m}\right) \delta_{4}-\hbar \mathcal{L}^{-1}\left\{\frac{(1-\alpha) s^{\alpha}+\alpha}{B(\alpha) s^{\alpha}} \mathcal{L}\left[\eta_{2} Q_{m-1}-\eta_{3} S_{m-1}\right]\right\}, \\
R_{m}(t)= & \left(\chi_{m}+\hbar\right) R_{m-1}-\hbar\left(1-\chi_{m}\right) \delta_{5}-\hbar \mathcal{L}^{-1}\left\{\frac{(1-\alpha) s^{\alpha}+\alpha}{B(\alpha) s^{\alpha}} \mathcal{L}\left[a R_{m-1}-\eta_{4} S_{m-1}\right]\right\},
\end{aligned}
$$

where

$$
\begin{aligned}
\mathrm{H}_{\mathrm{m}} & =\frac{1}{\Gamma(\mathrm{m}+1)}\left[\frac{\mathrm{d}^{\mathrm{m}}}{\mathrm{dq}^{\mathrm{m}}} \mathrm{N}\left[\left(\mathrm{q} \phi_{1}(\mathrm{t} ; \mathrm{q})\right)\left(\mathrm{q} \Phi_{3}(\mathrm{t} ; \mathrm{q})\right)\right]\right]_{\mathrm{q}=0^{\prime}} \\
\mathrm{K}_{\mathrm{m}}^{1} & =\frac{1}{\Gamma(\mathrm{m}+1)}\left[\frac{\mathrm{d}^{\mathrm{m}}}{\mathrm{dq^{m }}} \mathrm{N}\left[\left(\mathrm{q} \phi_{1}(\mathrm{t} ; \mathrm{q})\right)\left(\mathrm{q} \Phi_{2}(\mathrm{t} ; \mathrm{q})\right)\right]\right]_{\mathrm{q}=0^{\prime}} \\
\mathrm{K}_{\mathrm{m}}^{2} & =\frac{1}{\Gamma(\mathrm{m}+1)}\left[\frac{\mathrm{d}^{\mathrm{m}}}{\mathrm{dq^{m }}} \mathrm{N}\left[\left(\mathrm{q} \Phi_{2}(\mathrm{t} ; \mathrm{q})\right)\left(\mathrm{q} \Phi_{3}(\mathrm{t} ; \mathrm{q})\right)\right]\right]_{\mathrm{q}=0^{\prime}} \\
M_{\mathrm{m}} & =\frac{1}{\Gamma(\mathrm{m}+1)}\left[\frac{\mathrm{d}^{\mathrm{m}}}{\mathrm{dq}^{\mathrm{m}}} \mathrm{N}\left[\left(\mathrm{q} \Phi_{2}(\mathrm{t} ; \mathrm{q})\right)\left(\mathrm{q} \Phi_{3}(\mathrm{t} ; \mathrm{q})\right)\right]\right]_{\mathrm{q}=0}
\end{aligned}
$$

Taking the initial conditions and the iterative scheme (3.15), we obtain the following iterations

$$
\begin{aligned}
p^{0}: P_{0}(t) & =\delta_{1}+\frac{a(1-\alpha)}{B(\alpha)}+\frac{a \alpha t^{\alpha}}{B(\alpha) \Gamma(\alpha+1)}, \quad L_{0}(t)=\delta_{2}, \quad S_{0}(t)=\delta_{3}, \quad Q_{0}(t)=\delta_{4}, \quad R_{0}(t)=\delta_{5}, \\
p^{1}: P_{1}(t)= & m_{1}\left(\frac{(1-\alpha) \hbar t^{\alpha}}{\Gamma(\alpha+1)}+\frac{\alpha \hbar t^{2 \alpha}}{\Gamma(2 \alpha+1)}\right)+m_{2}\left((1-\alpha) \hbar+\frac{\alpha \hbar t^{\alpha}}{\Gamma(\alpha+1)}\right) \\
L_{1}(t)= & m_{3}\left((1-\alpha) \hbar+\frac{\alpha \hbar t^{\alpha}}{\Gamma(\alpha+1)}\right)-m_{4}\left(\frac{(1-\alpha) \hbar t^{\alpha}}{\Gamma(\alpha+1)}+\frac{\alpha \hbar t^{2 \alpha}}{\Gamma(2 \alpha+1)}\right), \\
S_{1}(t)= & m_{5}\left((1-\alpha) \hbar+\frac{\alpha \hbar t^{\alpha}}{\Gamma(\alpha+1)}\right), \\
Q_{1}(t)= & m_{6}\left((1-\alpha) \hbar+\frac{\alpha \hbar t^{\alpha}}{\Gamma(\alpha+1)}\right) \\
R_{1}(t)= & m_{7}\left((1-\alpha) \hbar+\frac{\alpha \hbar t^{\alpha}}{\Gamma(\alpha+1)}\right), \\
p^{2}: P_{2}(t)= & m_{1}\left[(1-\alpha) \frac{\hbar(1+\hbar) t^{\alpha}}{\Gamma(\alpha+1)}+\frac{\alpha \hbar(1+\hbar) t^{2 \alpha}}{\Gamma(2 \alpha+1)}\right]+m_{2}\left[(1-\alpha) \hbar(1+\hbar)+\frac{\alpha \hbar(1+\hbar) t^{\alpha}}{\Gamma(\alpha+1)}\right] \\
& +\frac{b(1-\alpha)}{B(\alpha)}\left[m_{5}\left(\delta_{1}+\frac{a(1-\alpha)}{B(\alpha)}+m_{2} \delta_{3}\right)+\frac{a m_{2}(1-\alpha)}{B(\alpha)}\right]\left((1-\alpha) \hbar^{2}+\frac{\alpha \hbar^{2} t^{\alpha}}{\Gamma(\alpha+1)}\right)
\end{aligned}
$$




$$
\begin{aligned}
& +\frac{b}{B(\alpha)}\left[m_{5}\left(\delta_{1}+\frac{a(1-\alpha)}{B(\alpha)}\right) \alpha+m_{5} \frac{a \alpha(1-\alpha)}{B(\alpha)}+m_{1} \delta_{3}(1-\alpha)+m_{2} \delta_{3} \alpha+\frac{a m_{1}(1-\alpha)}{B(\alpha) b}\right. \\
& \left.+\frac{a m_{2} \alpha}{B(\alpha) b}\right]\left(\frac{(1-\alpha) \hbar^{2} t^{\alpha}}{\Gamma(\alpha+1)}+\frac{\alpha \hbar^{2} t^{2 \alpha}}{\Gamma(2 \alpha+1)}\right) \\
& +\frac{b}{B(\alpha)}\left[\frac{m_{5} a \alpha^{2} \Gamma(2 \alpha+1)}{B(\alpha) \Gamma(\alpha+1) \Gamma(\alpha+1)}+m_{1} \alpha \delta_{3}+\frac{a m_{1} \alpha}{B(\alpha) b}\right]\left(\frac{(1-\alpha) \hbar^{2} t^{2 \alpha}}{\Gamma(2 \alpha+1)}+\frac{\alpha \hbar^{2} t^{3 \alpha}}{\Gamma(3 \alpha+1)}\right), \\
& \mathrm{L}_{2}(\mathrm{t})=\mathrm{m}_{3}\left((1-\alpha) \hbar(1+\hbar)+\frac{\alpha \hbar(1+\hbar) \mathrm{t}^{\alpha}}{\Gamma(\alpha+1)}\right)-\mathrm{m}_{4}\left((1-\alpha) \frac{\hbar(1+\hbar) \mathrm{t}^{\alpha}}{\Gamma(\alpha+1)}+\frac{\alpha \hbar(1+\hbar) \mathrm{t}^{2 \alpha}}{\Gamma(2 \alpha+1)}\right) \\
& +\frac{1}{B(\alpha)}\left[a_{3}(1-\alpha)-b(1-\alpha)\left(\left(\delta_{1}+\frac{a(1-\alpha)}{B(\alpha)}\right) m_{3}+\delta_{2}+m_{2}\right)\right. \\
& \left.+c(1-\alpha)\left(m_{3} \delta_{3}+m_{5} \delta_{2}\right)\right]\left((1-\alpha) \hbar^{2}+\frac{\alpha \hbar^{2} t^{\alpha}}{\Gamma(\alpha+1)}\right) \\
& -\frac{b}{B(\alpha)}\left[m_{3} \alpha\left(\delta_{1}+\frac{a(1-\alpha)}{B(\alpha)}\right)-m_{4}(1-\alpha)\left(\delta_{1}+\frac{a(1-\alpha)}{B(\alpha)}\right)+\frac{m_{3} \alpha a(1-\alpha)}{B(\alpha)}\right. \\
& \left.+\left(m_{1}(1-\alpha) \delta_{2}+m_{2} \alpha \delta_{2}\right)-a m_{3} \alpha+a m_{4}(1-\alpha)\right]\left(\frac{(1-\alpha) \hbar^{2} t^{\alpha}}{\Gamma(\alpha+1)}+\frac{\alpha \hbar^{2} t^{2 \alpha}}{\Gamma(2 \alpha+1)}\right) \\
& +\frac{b}{B(\alpha)}\left[m_{4} \alpha\left(\delta_{1}+\frac{a(1-\alpha)}{B(\alpha)}\right)-\frac{m_{3} a \alpha^{2}}{B(\alpha) \Gamma(\alpha+1)}+\frac{m_{4}(1-\alpha) a \alpha}{B(\alpha) \Gamma(\alpha+1)}-m_{1} \alpha+a m_{4} \alpha\right. \\
& \left.-c \delta_{3} \alpha m_{4}\right]\left(\frac{(1-\alpha) \hbar^{2} t^{2 \alpha}}{\Gamma(2 \alpha+1)}+\frac{\alpha \hbar^{2} t^{3 \alpha}}{\Gamma(3 \alpha+1)}\right)+\frac{a b m_{4} \alpha^{2}}{B(\alpha) \Gamma(\alpha+1)}\left(\frac{(1-\alpha) \hbar^{2} t^{3 \alpha}}{\Gamma(3 \alpha+1)}+\frac{\alpha \hbar^{2} t^{4 \alpha}}{\Gamma(4 \alpha+1)}\right), \\
& S_{2}(t)=m_{5}\left[(1-\alpha) \hbar(1+\hbar)+\frac{\alpha \hbar(1+\hbar) t^{\alpha}}{\Gamma(\alpha+1)}\right]+\frac{1}{B(\alpha)}\left[\eta_{1} m_{5}(1-\alpha)\right. \\
& \left.-c(1-\alpha)\left(m_{3} \delta_{3}+m_{5} \delta_{2}+f m_{6}(1-\alpha)\right)\right]\left((1-\alpha) \hbar^{2}+\frac{\alpha \hbar^{2} t^{\alpha}}{\Gamma(\alpha+1)}\right) \\
& -\frac{c}{B(\alpha)}\left[m_{3} \delta_{3} \alpha-m_{4} \delta_{3}(1-\alpha)+m_{5} \alpha \delta_{2}-\frac{\eta_{1} m_{5} \alpha}{c}+\frac{f m_{6} \alpha}{c}\right]\left(\frac{(1-\alpha) \hbar^{2} t^{\alpha}}{\Gamma(\alpha+1)}+\frac{\alpha \hbar^{2} t^{2 \alpha}}{\Gamma(\alpha+1)}\right) \\
& +c \delta_{3} \alpha m_{4}\left(\frac{(1-\alpha) \hbar^{2} t^{2 \alpha}}{\Gamma(2 \alpha+1)}+\frac{\alpha \hbar^{2} t^{3 \alpha}}{\Gamma(3 \alpha+1)}\right), \\
& \mathrm{Q}_{2}(\mathrm{t})=\mathrm{m}_{6}\left((1-\alpha) \hbar(1+\hbar)+\frac{\alpha \hbar(1+\hbar) \mathrm{t}^{\alpha}}{\Gamma(\alpha+1)}\right)+(1-\alpha)\left(\eta_{2} \mathrm{~m}_{6}-\eta_{3} \mathrm{~m}_{5}\right)\left((1-\alpha) \hbar^{2}+\frac{\alpha \hbar^{2} t^{\alpha}}{\Gamma(\alpha+1)}\right) \\
& +\alpha\left(\eta_{2} m_{6}-\eta_{3} m_{5}\right)\left(\frac{(1-\alpha) \hbar^{2} t^{\alpha}}{\Gamma(\alpha+1)}+\frac{\alpha \hbar^{2} t^{2 \alpha}}{\Gamma(2 \alpha+1)}\right), \\
& R_{2}(t)=m_{7}\left((1-\alpha) \hbar(1+\hbar)+\frac{\alpha \hbar(1+\hbar) t^{\alpha}}{\Gamma(\alpha+1)}\right)+(1-\alpha)\left(a m_{7}-\eta_{4} m_{5}\right)\left((1-\alpha) \hbar^{2}+\frac{\alpha \hbar^{2} t^{\alpha}}{\Gamma(\alpha+1)}\right) \\
& +\alpha\left(a m_{7}-\eta_{4} m_{5}\right)\left(\frac{(1-\alpha) \hbar^{2} t^{\alpha}}{\Gamma(\alpha+1)}+\frac{\alpha \hbar^{2} t^{2 \alpha}}{\Gamma(2 \alpha+1)}\right),
\end{aligned}
$$

where $m_{j}, j=1,2, \ldots, 7$, are given by

$$
\begin{array}{ll}
m_{1}=\frac{1}{B(\alpha)}\left(\alpha a^{2}+a b \delta_{3} \alpha\right), & m_{2}=\frac{1}{B(\alpha)}\left(a \delta_{1}+\frac{a^{2}(1-\alpha)}{B(\alpha)}+b \delta_{1} \delta_{3}+\frac{a b(1-\alpha)}{B(\alpha)}\right), \\
m_{3}=\frac{\delta_{2}}{B(\alpha)}\left(a-b\left(\delta_{1}+\frac{a(1-\alpha)}{B(\alpha)}\right)+c \delta_{3}\right), & m_{4}=\frac{a b \delta_{2} \alpha}{B(\alpha)}, \quad m_{5}=\frac{1}{B(\alpha)}\left(\eta_{1} \delta_{3}-c \delta_{2} \delta_{3}-f \delta_{4}\right), \\
m_{6}=\frac{1}{B(\alpha)}\left(\eta_{2} \delta_{4}-\eta_{3} \delta_{3}\right), & m_{7}=\frac{1}{B(\alpha)}\left(a \delta_{5}-\eta_{4} \delta_{3}\right) .
\end{array}
$$


Finally, the solutions of the system (3.12) are given by

$$
\begin{aligned}
& \mathrm{P}(\mathrm{t})=\mathrm{P}_{0}(\mathrm{t})+\mathrm{P}_{1}(\mathrm{t})+\mathrm{P}_{2}(\mathrm{t})+\cdots=\sum_{\mathrm{m}=0}^{\infty} \mathrm{P}_{\mathrm{m}}(\mathrm{t}), \\
& \mathrm{L}(\mathrm{t})=\mathrm{L}_{0}(\mathrm{t})+\mathrm{L}_{1}(\mathrm{t})+\mathrm{L}_{2}(\mathrm{t})+\cdots=\sum_{\mathrm{m}=0}^{\infty} \mathrm{L}_{\mathrm{m}}(\mathrm{t}), \\
& \mathrm{S}(\mathrm{t})=\mathrm{S}_{0}(\mathrm{t})+\mathrm{S}_{1}(\mathrm{t})+\mathrm{S}_{2}(\mathrm{t})+\cdots=\sum_{\mathrm{m}=0}^{\infty} \mathrm{S}_{\mathrm{m}}(\mathrm{t}), \\
& \mathrm{Q}(\mathrm{t})=\mathrm{Q}_{0}(\mathrm{t})+\mathrm{Q}_{1}(\mathrm{t})+\mathrm{Q}_{2}(\mathrm{t})+\cdots=\sum_{\mathrm{m}=0}^{\infty} \mathrm{Q}_{\mathrm{m}}(\mathrm{t}), \\
& \mathrm{R}(\mathrm{t})=\mathrm{R}_{0}(\mathrm{t})+\mathrm{R}_{1}(\mathrm{t})+\mathrm{R}_{2}(\mathrm{t})+\cdots=\sum_{\mathrm{m}=0}^{\infty} \mathrm{R}_{\mathrm{m}}(\mathrm{t}) .
\end{aligned}
$$

Another solution of model (3.12) can be obtained involving Eq. (2.4). In the Atangana-Baleanu sense, system (3.12) is equivalent to Volterra type. The following iterative scheme converges to the exact solution taking the limit for a large value of $n$

$$
\begin{aligned}
P_{(n+1)}(t)= & \frac{1-\alpha}{B(\alpha)}\left\{a-a P_{n}(t)-b P_{n}(t) S_{n}(t)\right\} \\
& +\frac{\alpha}{B(\alpha) \Gamma(\alpha)} \int_{0}^{t}(t-\tau)^{\alpha-1}\left\{a-a P_{n}(\tau)-b P_{n}(\tau) S_{n}(\tau)\right\} d \tau, \\
L_{(n+1)}(t)= & \frac{1-\alpha}{B(\alpha)}\left\{-a L_{n}(t)+b P_{n}(t) L_{n}(t)-c L_{n}(t) S_{n}(t)\right\} \\
& +\frac{\alpha}{B(\alpha) \Gamma(\alpha)} \int_{0}^{t}(t-\tau)^{\alpha-1}\left\{-a L_{n}(\tau)+b P_{n}(\tau) L_{n}(\tau)-c L_{n}(\tau) S_{n}(\tau)\right\} d \tau, \\
S_{(n+1)}(t)= & \frac{1-\alpha}{B(\alpha)}\left\{-\eta_{1} S_{n}(t)+c L_{n}(t) S_{n}(t)+f Q_{n}(t)\right\} \\
& +\frac{\alpha}{B(\alpha) \Gamma(\alpha)} \int_{0}^{t}(t-\tau)^{\alpha-1}\left\{-\eta_{1} S_{n}(\tau)+c L_{n}(\tau) S_{n}(\tau)+f Q_{n}(\tau)\right\} d \tau, \\
Q_{(n+1)}(t)= & \frac{1-\alpha}{B(\alpha)}\left\{-\eta_{2} Q_{n}(t)+\eta_{3} S_{n}(t)\right\}+\frac{\alpha}{B(\alpha) \Gamma(\alpha)} \int_{0}^{t}(t-\tau)^{\alpha-1}\left\{-\eta_{2} Q_{n}(\tau)+\eta_{3} S_{n}(\tau)\right\} d \tau, \\
R_{n}(t)= & \frac{1-\alpha}{B(\alpha)}\left\{-a R_{n}(t)+\eta_{4} S_{n}(t)\right\}+\frac{\alpha}{B(\alpha) \Gamma(\alpha)} \int_{0}^{t}(t-\tau)^{\alpha-1}\left\{-a R_{n}(\tau)+\eta_{4} S_{n}(\tau)\right\} d \tau .
\end{aligned}
$$

Theorem 3.2. We demonstrate the existence of the solution using Picard-Lindelof approach.

Proof. We consider the following operator

$$
\begin{aligned}
& \Xi_{1}(t, \zeta)=a-a P(t)-b P(t) S(t), \\
& \Xi_{2}(t, \zeta)=-a L(t)+b P(t) L(t)-c L(t) S(t), \\
& \Xi_{3}(t, \zeta)=-\eta_{1} S(t)+c L(t) S(t)+f Q(t), \\
& \Xi_{4}(t, \zeta)=-\eta_{2} Q(t)+\eta_{3} S(t), \\
& \Xi_{5}(t, \zeta)=-a R(t)+\eta_{4} S(t),
\end{aligned}
$$

where $\Xi_{1}(t, \zeta), \Xi_{2}(t, \zeta), \Xi_{3}(t, \zeta), \Xi_{4}(t, \zeta)$, and $\Xi_{5}(t, \zeta)$ are contraction respect to $\theta, \rho, v$, $\phi$, and $\sigma$ for the first, second, third, fourth, and fifth functions, respectively. 
Let

where,

$$
\begin{aligned}
& \Omega_{1}=\sup \left\|\gamma_{\gamma_{\epsilon, \kappa_{1}}} \Xi_{1}(\mathrm{t}, \zeta)\right\| ; \quad \Omega_{2}=\sup \|\|_{\gamma_{\epsilon, \kappa_{2}}} \Xi_{2}(\mathrm{t}, \zeta)\left\|; \quad \Omega_{3}=\sup \right\|_{\gamma_{\epsilon, \kappa_{3}}} \Xi_{3}(\mathrm{t}, \zeta) \| ; \\
& \Omega_{4}=\sup \left\|\Upsilon_{\epsilon, \kappa_{4}} \Xi_{4}(t, \zeta)\right\| ; \quad \Omega_{5}=\sup \left\|\gamma_{\epsilon, \kappa_{5}} \Xi_{5}(t, \zeta)\right\| \text {, }
\end{aligned}
$$

$$
\begin{array}{ll}
\Upsilon_{\epsilon, \kappa_{1}}=|t-a, t+a| \times\left[\theta-b_{1}, \theta+b_{1}\right]=\epsilon_{1} \times \kappa_{1}, & \Upsilon_{\epsilon, \kappa_{2}}=|t-a, t+a| \times\left[\rho-\kappa_{2}, \rho+\kappa_{2}\right]=\epsilon_{1} \times \kappa_{2}, \\
\Upsilon_{\epsilon, \kappa_{3}}=|t-a, t+a| \times\left[v-\kappa_{3}, v+\kappa_{3}\right]=\epsilon_{1} \times \kappa_{3}, & \Upsilon_{\epsilon, \kappa_{4}}=|t-a, t+a| \times\left[\phi-\kappa_{4}, \phi+\kappa_{4}\right]=\epsilon_{1} \times \kappa_{4}, \\
\Upsilon_{\epsilon, \kappa_{5}}=|t-a, t+a| \times\left[\sigma-\kappa_{5}, \sigma+\kappa_{5}\right]=\epsilon_{1} \times \kappa_{5} . &
\end{array}
$$

Considering the Picard's operator, we have

$$
\Theta: \Upsilon\left(\epsilon_{1}, \mathrm{k}_{1}, \mathrm{~K}_{2}, \mathrm{~K}_{3}, \mathrm{~K}_{4}, \mathrm{~K}_{5}\right) \rightarrow \Upsilon\left(\epsilon_{1}, \mathrm{~K}_{1}, \mathrm{~K}_{2}, \mathrm{~K}_{3}, \mathrm{~K}_{4}, \mathrm{~K}_{5}\right),
$$

defined as follows

$$
\Theta \Omega(t)=\Omega_{0}(t)+\Delta(t, \Omega(t)) \frac{1-\alpha}{B(\alpha)}+\frac{\alpha}{B(\alpha) \Gamma(\alpha)} \int_{0}^{t}(t-\tau)^{\alpha-1} \Delta(\tau, \Omega(\tau)) d \tau,
$$

where $\Omega(t)=\{P(t), L(t), S(t), Q(t), R(t)\}, \Omega_{0}(t)=\left\{g_{1}, g_{2}, g_{3}, g_{4}, g_{5}\right\}$, and

$$
\Delta(t, \Omega(t))=\left\{\Xi_{1}(t, \theta(t)), \Xi_{2}(t, \rho(t)), \Xi_{3}(t, v(t)), \Xi_{4}(t, \phi(t)), \Xi_{5}(t, \sigma(t))\right\} .
$$

Now we assume that all the solutions are bounded within a period of time

$$
\begin{aligned}
& \|\Omega(t)\|_{\infty} \leqslant \max \left\{\kappa_{1}, \kappa_{2}, \kappa_{3}, \kappa_{4}, \kappa_{5}\right\}, \\
& \left\|\Omega(t)-\Omega_{0}(t)\right\|=\left\|\Delta(t, \Omega(t)) \frac{1-\alpha}{B(\alpha)}+\frac{\alpha}{B(\alpha) \Gamma(\alpha)} \int_{0}^{t}(t-\tau)^{\alpha-1} \Delta(\tau, \Omega(\tau)) d \tau\right\| \\
& \leqslant \frac{1-\alpha}{\mathrm{B}(\alpha)}\|\Delta(\mathrm{t}, \Omega(\mathrm{t}))\|+\frac{\alpha}{\mathrm{B}(\alpha) \Gamma(\alpha)} \int_{0}^{\mathrm{t}}(\mathrm{t}-\tau)^{\alpha-1}\|\Delta(\tau, \Omega(\tau))\| \mathrm{d} \tau \\
& \leqslant \frac{1-\alpha}{\mathrm{B}(\alpha)} \chi=\max \left\{\mathrm{\kappa}_{1}, \mathrm{\kappa}_{2}, \mathrm{\kappa}_{3}, \mathrm{\kappa}_{4}, \mathrm{\kappa}_{5}\right\}+\frac{\alpha}{\mathrm{B}(\alpha)} \xi \vartheta^{\alpha} \leqslant \vartheta \xi \leqslant \kappa=\max \left\{\mathrm{\kappa}_{1}, \mathrm{\kappa}_{2}, \mathrm{\kappa}_{3}, \mathrm{\kappa}_{4}, \mathrm{\kappa}_{5}\right\} .
\end{aligned}
$$

Here we request that

$$
\vartheta<\frac{\kappa}{\xi}
$$

Using the fixed point theorem of Banach space together with the metric, we have

$$
\begin{aligned}
\left\|\Theta \Omega_{1}-\Theta \Omega_{2}\right\|_{\infty}= & \sup \|_{t \in \epsilon}\left|\Omega_{1}-\Omega_{2}\right|, \\
\left\|\Theta \Omega_{1}-\Theta \Omega_{2}\right\|= & \|\left\{\Delta\left(t, \Omega_{1}(t)\right)-\Delta\left(t, \Omega_{2}(t)\right)\right\} \frac{1-\alpha}{B(\alpha)} \\
& +\frac{\alpha}{B(\alpha) \Gamma(\alpha)} \int_{0}^{t}(t-\tau)^{\alpha-1}\left\{\Delta\left(\tau, \Omega_{1}(t)\right)-\Delta\left(\tau, \Omega_{2}(t)\right)\right\} d \tau \|, \\
\leqslant & \frac{1-\alpha}{B(\alpha)}\left\|\Delta\left(t, \Omega_{1}(t)\right)-\Delta\left(t, \Omega_{2}(t)\right)\right\|+\frac{\alpha}{B(\alpha) \Gamma(\alpha)} \int_{0}^{t}(t-\tau)^{\alpha-1}\left\|\Delta\left(\tau, \Omega_{1}(t)\right)-\Delta\left(\tau, \Omega_{2}(t)\right)\right\| d \tau, \\
\leqslant & \left.\left.\frac{1-\alpha}{B(\alpha)} \omega\left\|\Omega_{1}(t)-\Omega_{2}(t)\right\|+\frac{\alpha \omega}{B(\alpha) \Gamma(\alpha)} \int_{0}^{t}(t-\tau)^{\alpha-1} \| \Omega_{1}(t)\right)-\Omega_{2}(t)\right) \| d \tau, \\
\leqslant & \left.\left.\left\{\frac{1-\alpha}{B(\alpha)} \omega+\frac{\alpha \omega \vartheta}{B(\alpha) \Gamma(\alpha)}\right\} \| \Omega_{1}(t)\right)-\Omega_{2}(t)\right) \| d \tau, \\
\leqslant & \left.\left.\vartheta \omega \| \Omega_{1}(t)\right)-\Omega_{2}(t)\right) \|,
\end{aligned}
$$

with $\omega<1$. Since $\Omega$ is a contraction we have that $\vartheta \omega<1$, thus the defined operator $\Theta$ is a contraction too. We conclude that the system (3.17) as a unique set of solution.

The numerical approximation of Atangana-Baleanu fractional integral (2.4) using the Adams-Moulton rule is given by [2] 


$$
{ }_{0}^{A B} \mathcal{J}_{t}^{\alpha}\left[f\left(t_{n+1}\right)\right]=\frac{1-\alpha}{B(\alpha)}\left[\frac{f\left(t_{n+1}\right)-f\left(t_{n}\right)}{2}\right]+\frac{\alpha}{\Gamma(\alpha)} \sum_{k=0}^{\infty}\left[\frac{f\left(t_{k+1}\right)-f\left(t_{k}\right)}{2}\right] b_{k}^{\alpha},
$$

where $b_{k}^{\alpha}=(k+1)^{1-\alpha}-(k)^{1-\alpha}$.

Using the above numerical scheme, we have

$$
\begin{aligned}
& P_{(n+1)}(t)-P_{(n)}(t)=P_{0}^{n}(t)+\left\{\frac { 1 - \alpha } { B ( \alpha ) } \left[a-a\left(\frac{P_{(n+1)}(t)-P_{(n)}(t)}{2}\right)-b\left(\frac{P_{(n+1)}(t)-P_{(n)}(t)}{2}\right)\right.\right. \\
& \left.\left.\cdot\left(\frac{S_{(n+1)}(t)-S_{(n)}(t)}{2}\right)\right]\right\}+\frac{\alpha}{B(\alpha)} \sum_{k=0}^{\infty}(k+1)^{1-\alpha}-(k)^{1-\alpha}[a \\
& \left.-a\left(\frac{P_{(k+1)}(t)-P_{(k)}(t)}{2}\right)-b\left(\frac{P_{(k+1)}(t)-P_{(k)}(t)}{2}\right)\left(\frac{S_{(k+1)}(t)-S_{(k)}(t)}{2}\right)\right] \text {, } \\
& \mathrm{L}_{(\mathrm{n}+1)}(\mathrm{t})-\mathrm{L}_{(\mathrm{n})}(\mathrm{t})=\mathrm{L}_{0}^{\mathrm{n}}(\mathrm{t})+\left\{\frac { 1 - \alpha } { \mathrm { B } ( \alpha ) } \left[-\mathrm{a}\left(\frac{\mathrm{L}_{(\mathrm{n}+1)}(\mathrm{t})-\mathrm{L}_{(\mathrm{n})}(\mathrm{t})}{2}\right)+\mathrm{b}\left(\frac{\mathrm{P}_{(\mathrm{n}+1)}(\mathrm{t})-\mathrm{P}_{(\mathrm{n})}(\mathrm{t})}{2}\right)\right.\right. \\
& \left.\left.\cdot\left(\frac{\mathrm{L}_{(\mathrm{n}+1)}(\mathrm{t})-\mathrm{L}_{(\mathrm{n})}(\mathrm{t})}{2}\right)-c\left(\frac{\mathrm{L}_{(\mathrm{n}+1)}(\mathrm{t})-\mathrm{L}_{(\mathrm{n})}(\mathrm{t})}{2}\right)\left(\frac{\mathrm{S}_{(\mathrm{n}+1)}(\mathrm{t})-\mathrm{S}_{(\mathrm{n})}(\mathrm{t})}{2}\right)\right]\right\} \\
& +\frac{\alpha}{\mathrm{B}(\alpha)} \sum_{\mathrm{k}=0}^{\infty}(\mathrm{k}+1)^{1-\alpha}-(\mathrm{k})^{1-\alpha}\left[-\mathrm{a}\left(\frac{\mathrm{L}_{(\mathrm{k}+1)}(\mathrm{t})-\mathrm{L}_{(\mathrm{k})}(\mathrm{t})}{2}\right)+\mathrm{b}\left(\frac{\mathrm{P}_{(\mathrm{k}+1)}(\mathrm{t})-\mathrm{P}_{(\mathrm{k})}(\mathrm{t})}{2}\right)\right. \\
& \left.\cdot\left(\frac{\mathrm{L}_{(\mathrm{k}+1)}(\mathrm{t})-\mathrm{L}_{(\mathrm{k})}(\mathrm{t})}{2}\right)-c\left(\frac{\mathrm{L}_{(\mathrm{k}+1)}(\mathrm{t})-\mathrm{L}_{(\mathrm{k})}(\mathrm{t})}{2}\right)\left(\frac{\mathrm{S}_{(\mathrm{k}+1)}(\mathrm{t})-\mathrm{S}_{(\mathrm{k})}(\mathrm{t})}{2}\right)\right] \text {, } \\
& S_{(n+1)}(t)-S_{(n)}(t)=S_{0}^{n}(t)+\left\{\frac { 1 - \alpha } { B ( \alpha ) } \left[-\eta_{1}\left(\frac{S_{(n+1)}(t)-S_{(n)}(t)}{2}\right)+c\left(\frac{L_{(n+1)}(t)-L_{(n)}(t)}{2}\right)\right.\right. \\
& \left.\left.\cdot\left(\frac{S_{(n+1)}(t)-S_{(n)}(t)}{2}\right)+f\left(\frac{Q_{(n+1)}(t)-Q_{(n)}(t)}{2}\right)\right]\right\} \\
& +\frac{\alpha}{B(\alpha)} \sum_{k=0}^{\infty}(k+1)^{1-\alpha}-(k)^{1-\alpha}\left[-\eta_{1}\left(\frac{S_{(k+1)}(t)-S_{(k)}(t)}{2}\right)\right. \\
& +c\left(\frac{\mathrm{L}_{(\mathrm{k}+1)}(\mathrm{t})-\mathrm{L}_{(\mathrm{k})}(\mathrm{t})}{2}\right) \\
& \left.\cdot\left(\frac{S_{(k+1)}(t)-S_{(k)}(t)}{2}\right)+f\left(\frac{Q_{(k+1)}(t)-Q_{(k)}(t)}{2}\right)\right] \text {, } \\
& \mathrm{Q}_{(\mathrm{n}+1)}(\mathrm{t})-\mathrm{Q}_{(\mathrm{n})}(\mathrm{t})=\mathrm{Q}_{0}^{\mathrm{n}}(\mathrm{t})+\left\{\frac{1-\alpha}{\mathrm{B}(\alpha)}\left[-\eta_{2}\left(\frac{\mathrm{Q}_{(\mathrm{n}+1)}(\mathrm{t})-\mathrm{Q}_{(\mathrm{n})}(\mathrm{t})}{2}\right)+\eta_{3}\left(\frac{\mathrm{S}_{(\mathrm{n}+1)}(\mathrm{t})-\mathrm{S}_{(\mathrm{n})}(\mathrm{t})}{2}\right)\right]\right\} \\
& +\frac{\alpha}{B(\alpha)} \sum_{k=0}^{\infty}(k+1)^{1-\alpha}-(k)^{1-\alpha}\left[-\eta_{2}\left(\frac{Q_{(k+1)}(t)-Q_{(k)}(t)}{2}\right)\right. \\
& \left.+\eta_{3}\left(\frac{S_{(k+1)}(t)-S_{(k)}(t)}{2}\right)\right] \\
& R_{(n+1)}(t)-R_{(n)}(t)=R_{0}^{n}(t)+\left\{\frac{1-\alpha}{B(\alpha)}\left[-a\left(\frac{R_{(n+1)}(t)-R_{(n)}(t)}{2}\right)+\eta_{4}\left(\frac{S_{(n+1)}(t)-S_{(n)}(t)}{2}\right)\right]\right\} \\
& +\frac{\alpha}{B(\alpha)} \sum_{k=0}^{\infty}(k+1)^{1-\alpha}-(k)^{1-\alpha}\left[-a\left(\frac{R_{(k+1)}(t)-R_{(k)}(t)}{2}\right)\right. \\
& \left.+\eta_{4}\left(\frac{S_{(k+1)}(t)-S_{(k)}(t)}{2}\right)\right] \text {. }
\end{aligned}
$$




\section{Numerical results and discussion}

In this section, we present numerical simulations of the model given by Eq. (1.1) in the LiouvilleCaputo and Atangana-Baleanu-Caputyo sense. We utilize the values of the parameters $a=0.04, b=0.23$, $c=0.3, d=0.2, e=0.4$, and $f=0.25$. The initial conditions are given by $P(0)=0.60301, L(0)=0.24000$, $\mathrm{S}(0)=0.10628, \mathrm{Q}(0)=0.03260$, and $\mathrm{R}(0)=0.0 .01811$. The simulation time is 500 seconds and the step size used in evaluating the approximate solutions was $h=0.005$. Figs $1 \mathrm{a}-2 \mathrm{~d}$ show the numerical solutions (3.7) and (3.16) obtained by application of the MHATM through a graphical representation.

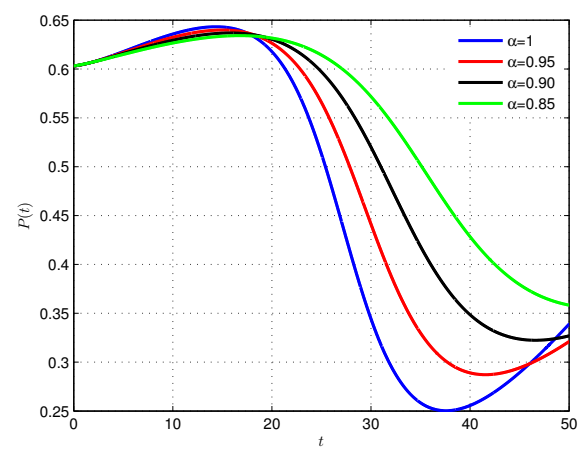

(a)

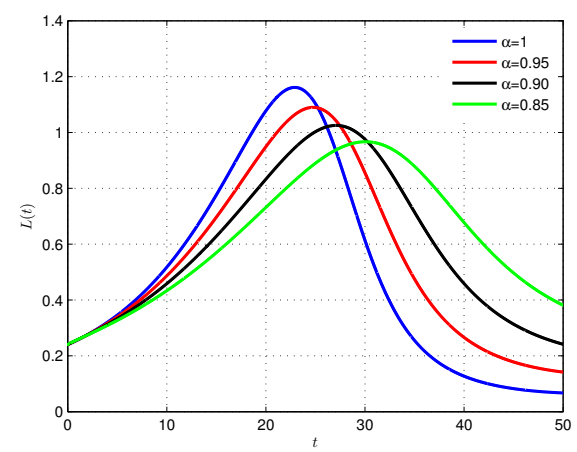

(c)

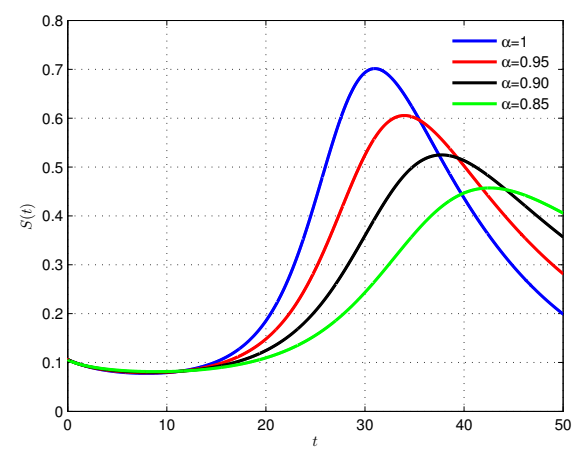

(e)

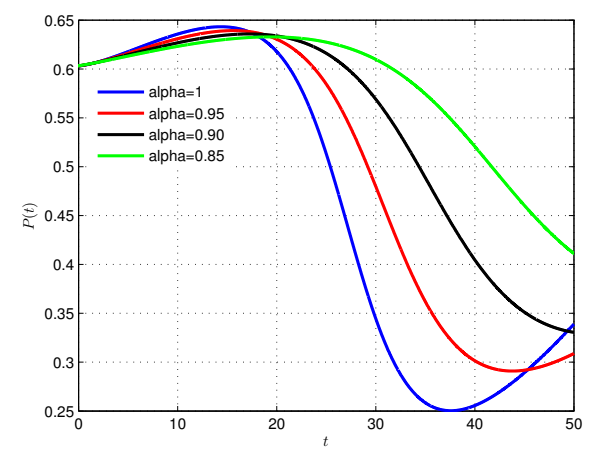

(b)

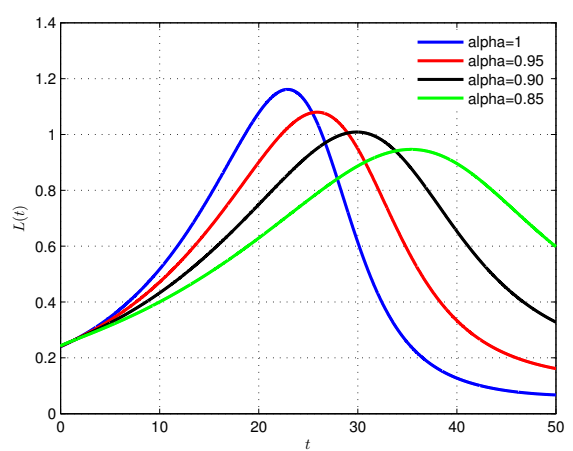

$(\mathrm{d})$

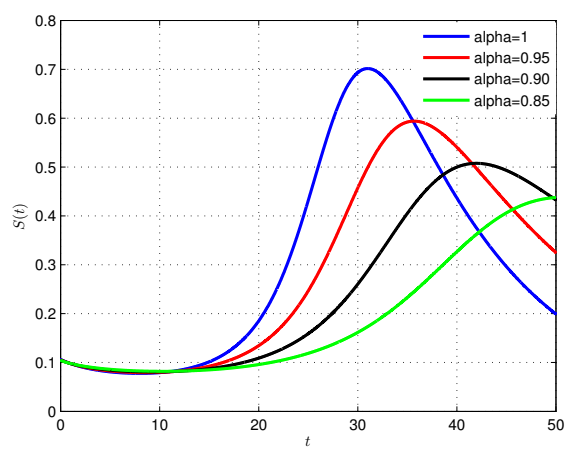

(f)

Figure 1: Numerical simulation for the potential smokers $P(t)$, ocassional smokers $L(t)$ and heavy smokers $S(t)$ vs. time $t$ in days for distinct values of $\alpha$ arbitrary chosen. In (a), (c) and (e) numerical results obtained with Liouville-Caputo fractional-order derivative. In (b), (d) and (f) numerical results obtained with Atangana-Baleanu-Caputo fractional-order derivative. 


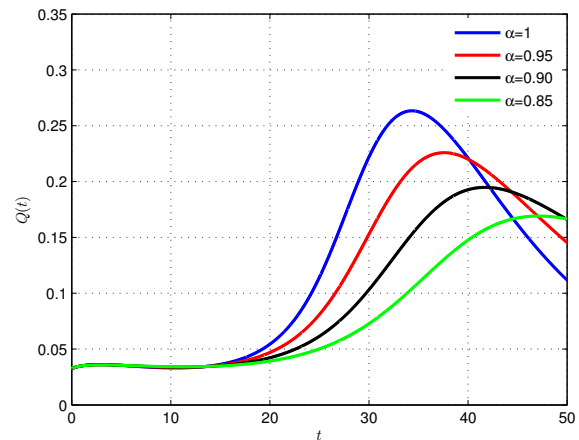

(a)

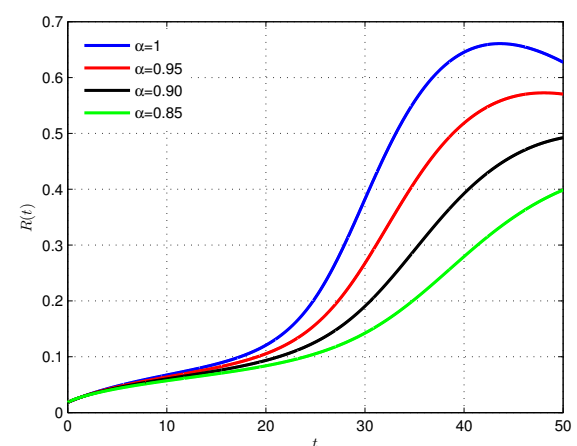

(c)

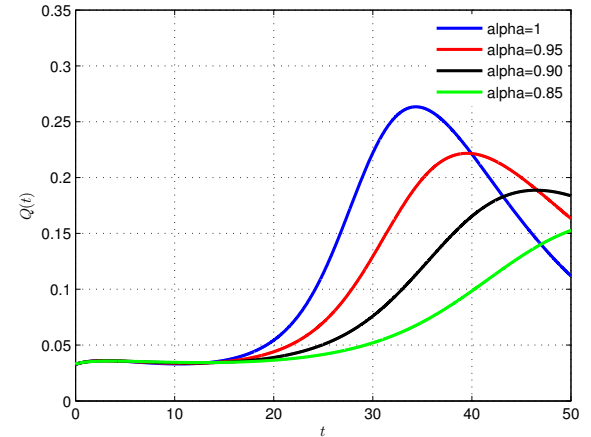

(b)

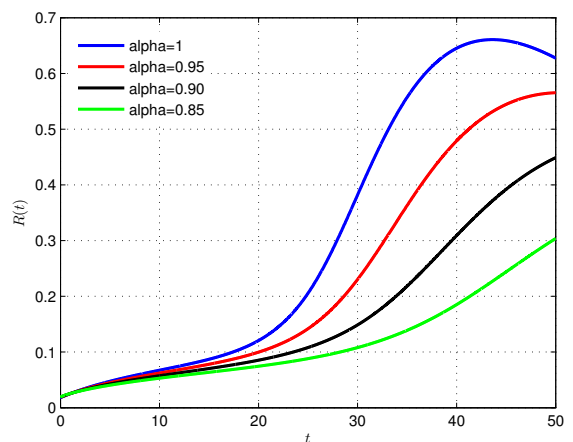

(d)

Figure 2: Numerical simulation for the temporary quiters $Q(t)$ and permanent quitters $R(t)$ vs. time $t$ in days for distinct values of $\alpha$ arbitrary chosen. In (a) and (c) numerical results obtained with LiouvilleCaputo fractional-order derivative. In (b) and (d) numerical results obtained with Atangana-BaleanuCaputo fractional-order derivative.

Figs 3a-4d show the phase diagram and the chaotic trajectories of the fractional-order smoking model via Liouville-Caputo and atangana-Baleanu-Caputo fractional-order derivatives. The numerical simulation has carried out using MATLAB ${ }^{\circledR}$ subroutines written based on Eqs. (3.11) and (3.18) for the commensurate order $\alpha=0.90$. The simulation time is 500 seconds and the step size used in evaluating the approximate solutions was $h=0.005$.

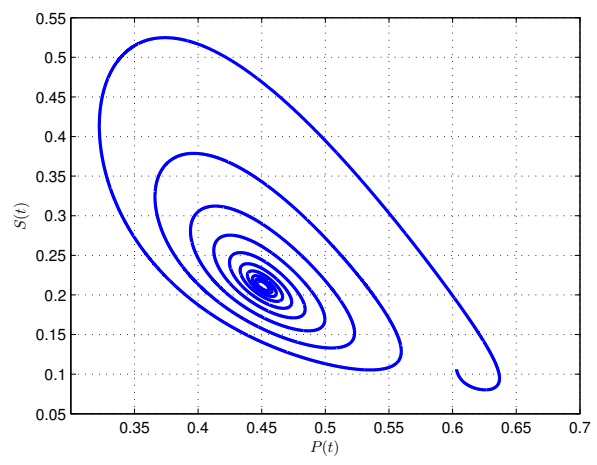

(a)

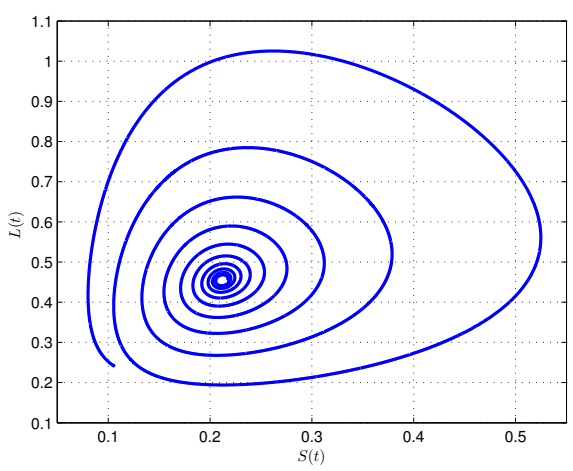

(b) 


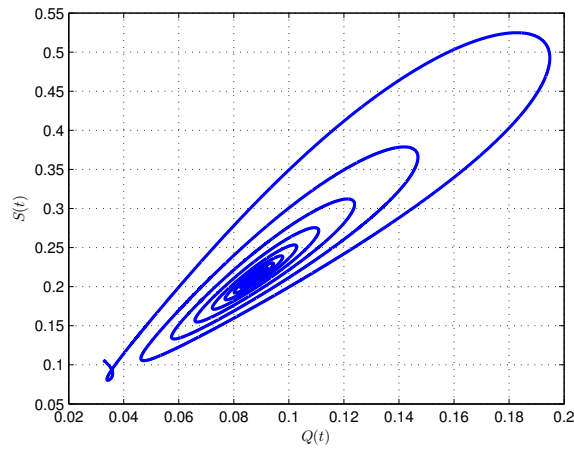

(c)

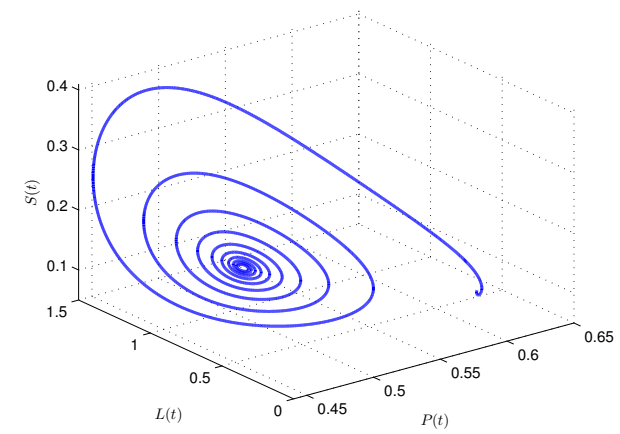

(d)

Figure 3: Phase portraits and chaotic trajectories of the fractional-order smoking model via LiouvilleCaputo fractional-order derivative. In (a) Potential smokers $P(t)$ vs. Heavy smokers $S(t)$. In (b) Heavy smokers $S(t)$ vs. Ocassional smokers $L(t)$. In (c) Temporary quiters vs. Heavy smokers $S(t)$. In $(d)$ Potential smokers $P(t)$ vs. Ocassional smokers $L(t)$ vs. Heavy smokers $S(t)$ for $\alpha=0.90$ arbitrary chosen.

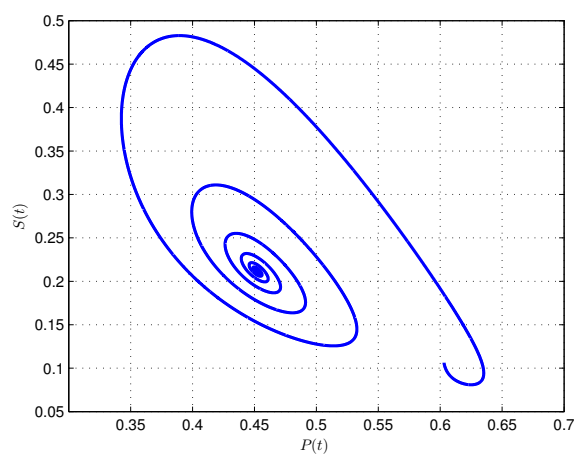

(a)

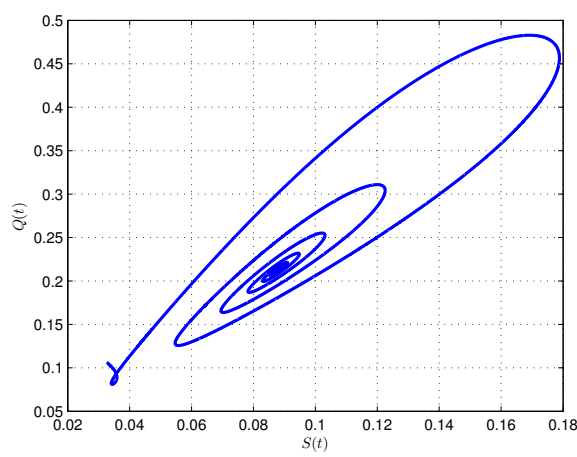

(c)

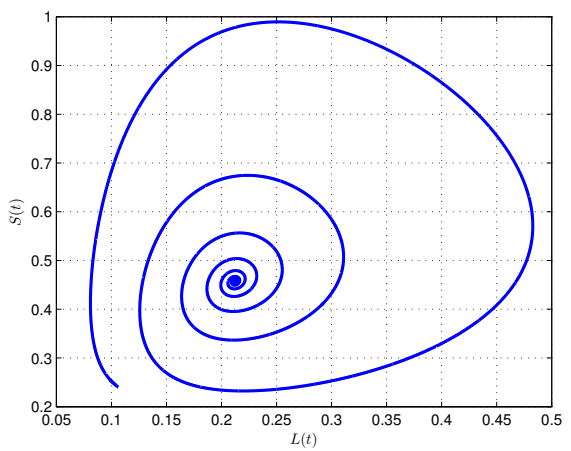

(b)

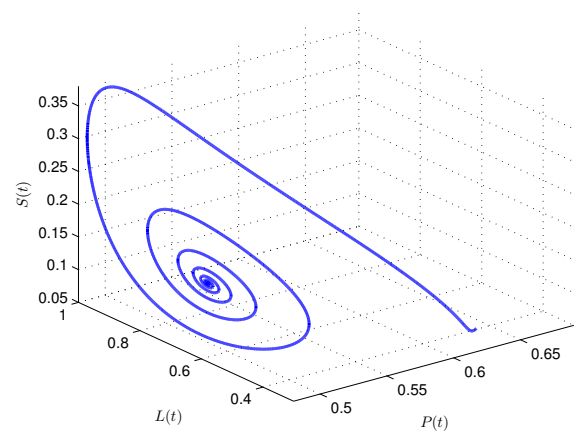

(d)

Figure 4: Phase portraits and chaotic trajectories of the fractional-order smoking model via AtanganaBaleanu-Caputo fractional-order derivative. In (a) Potential smokers $P(t)$ vs. Heavy smokers $S(t)$. In (b) Heavy smokers $S(t)$ vs. Ocassional smokers $L(t)$. In (c) Temporary quiters vs. Heavy smokers $S(t)$. In $(d)$ Potential smokers $P(t)$ vs. Ocassional smokers $L(t)$ vs. Heavy smokers $S(t)$ for $\alpha=0.90$ arbitrary chosen.

It is worth noting that, the prediction depends on the value of the fractional-order also beside the theoretical parameters. The numerical results showed that the approximate solutions at different values 
of $\alpha$ are close to the values of the exact solution with high accuracy and the accuracy increases as the order of approximation increases. In this sense, the parameter $\alpha$ plays a crucial role in the numerical solution and it can be used to find new insights of the generalized biological models.

\section{Conclusion}

This work addresses a new mathematical model able to describe the smoking dynamic with the Liouville-Caputo and Atangana-Baleanu fractional-order derivatives. The mathematical model is built using a power law and the new fractional differentiation that involve the generalized Mittag-Leffler function as kernel due to the non-locality of the model. To demonstrate the existence and uniqueness of a system of solutions the fixed point theorem and Picard-Lindelof approach are discussed. Analytical approximate solutions were obtained with the MHATM for the smoking model with two different fractional derivatives. To present the effect of fractional order some numerical simulations are performed. These Figures shown that the Liouville-Caputo fractional derivative is more affected by the past compared with the new fractional operator based in the Mittag-Leffler function which shows a rapid stabilization. The computer used for obtaining the results in this paper is an Intel Core i7, 2.6 GHz processor, 16.0-GB RAM (Matlab R.2013a).

\section{Acknowledgment}

José Francisco Gómez Aguilar acknowledges the support provided by CONACYT: cátedras CONACYT para jóvenes investigadores 2014. J. F. Gómez Aguilar, R. F. Escobar Jiménez, M. A. Taneco Hernández, and V. H. Olivares Peregrino acknowledge the support provided by SNI-CONACyT.

\section{References}

[1] O. J. Algahtani, A. Zeb, G. Zaman, S. Momani, I. H. Jung, Mathematical study of smoking model by incorporating campaign class, Wulfenia, 22 (2015), 205-216. 1

[2] B. S. T. Alkahtani, Chua's circuit model with Atangana-Baleanu derivative with fractional order, Chaos Solitons Fractals, 89 (2016), 547-551. 3.2

[3] A. Atangana, D. Baleanu, New Fractional Derivatives with Nonlocal and Non-Singular Kernel: Theory and Application to Heat Transfer Model, Therm Sci., 20 (2016), 763-769. 2

[4] A. Atangana, I. Koca, Model of Thin Viscous Fluid Sheet Flow within the Scope of Fractional Calculus: Fractional Derivative with and No Singular Kernel, Fund. Inform., 151 (2017), 145-159. 1

[5] C. Castillo-Garsow, G. Jordan-Salivia, A. R. Herrera, Mathematical models for the dynamics of tobacco use, recovery, and relapse, Technical Report Series BU-1505-M, Cornell University, Ithaca, (1997). 1

[6] J. S. Choi, D. Kumar, J. Singh, R. Swroop, Analytical techniques for system of time fractional nonlinear differential equations, J. Korean Math. Soc., 54 (2017), 1209-1229. 1

[7] K. Diethelm, N. J. Ford, A. D. Freed, Detailed error analysis for a fractional Adams method, Numer. Algorithms, 36 (2004), 31-52. 3.1

[8] Q. Din, M. Ozair, T. Hussain, U. Saeed, Qualitative behavior of a smoking model, Adv. Difference Equ., 2016 (2016), 12 pages. 1

[9] V. S. Ertürk, G. Zaman, S. Momani, A numeric-analytic method for approximating a giving up smoking model containing fractional derivatives, Comput. Math. Appl., 64 (2012), 3065-3074. 1

[10] F. Haq, K. Shah, G. ur Rahman, M. Shahzad, Numerical solution of fractional order smoking model via laplace Adomian decomposition method, Alexandria Engineering Journal, 2017 (2017), 9 pages. 1

[11] J. Hristov, Integral balance solutions to applied models involving time-fractional derivatives-The scope of the method and results thereof, Commun. Frac. Calc., 4 (2013), 64-104. 1

[12] J. Hristov, Integral-Balance Solution to Nonlinear Subdiffusion Equation, Frontiers in Fractional Calculus, 2017 (2017), 71-106. 1

[13] S. Kumar, A new analytical modelling for telegraph equation via Laplace transform, Appl. Math. Model., 38 (2014), 3154-3163. 3.1

[14] S. Kumar, A. Kumar, I. K. Argyros, A new analysis for the Keller-Segel model of fractional order, Numer. Algorithms, 75 (2017), 213-228. 1, 3.1, 3.2 
[15] S. Kumar, M. M. Rashidi, New analytical method for gas dynamic equation arising in shock fronts, Comput. Phys. Commun., 185 (2014), 1947-1954. 3.1

[16] D. Kumar, J. Singh, M. M. Al Qurashi, D. Baleanu, Analysis of logistic equation pertaining to a new fractional derivative with non-singular kernel, Adv. Mech. Eng., 9 (2017), 8 pages. 1

[17] D. Kumar, J. Singh, D. Baleanu, A new numerical algorithm for fractional Fitzhugh-Nagumo equation arising in transmission of nerve impulses, Nonlinear Dynam., 91 (2018), 307-317.

[18] D. Kumar, J. Singh, D. Baleanu, A new analysis of the Fornberg-Whitham equation pertaining to a fractional derivative with Mittag-Leffler-type kernel, D. Eur. Phys. J. Plus, 133 (2018), 10 pages.

[19] D. Kumar, J. Singh, D. Baleanu, Sushila, Analysis of regularized long-wave equation associated with a new fractional operator with Mittag-Leffler type kernel, Phys. A, 492 (2018), 155-167. 1

[20] C. P. Li, C. X. Tao, On the fractional Adams method, Comput. Math. Appl., 58 (2009), 1573-1588. 3.1

[21] Z. Odibat, A. S. Bataineh, An adaptation of homotopy analysis method for reliable treatment of strongly nonlinear problems: construction of homotopy polynomials, Math. Meth. Appl. Sci., 38 (2015), 991-1000. 3.1

[22] K. M. Owolabi, Mathematical analysis and numerical simulation of chaotic noninteger order differential systems with Riemann-Liouville derivative, Numer. Methods Partial Differential Equations, 34 (2018), 274-295. 1

[23] K. M. Owolabi, Mathematical modelling and analysis of two-component system with Caputo fractional derivative order, Chaos Solitons Fractals, 103 (2017), 544-554.

[24] K. M. Owolabi, Robust and adaptive techniques for numerical simulation of nonlinear partial differential equations of fractional order, Commun. Nonlinear Sci. Numer. Simul., 44 (2017), 304-317.

[25] K. M. Owolabi, A. Atangana, Numerical approximation of nonlinear fractional parabolic differential equations with Caputo-Fabrizio derivative in Riemann-Liouville sense, Chaos Solitons Fractals, 99 (2017), 171-179.

[26] K. M. Owolabi, A. Atangana, Numerical Simulation of Noninteger Order System in Subdiffusive, Diffusive, and Superdiffusive Scenarios, J. Comput. Nonlinear Dynam, 12 (2017), 7 pages.

[27] I. Podlubny, Fractional Differential Equations, Academic Press, San Diego, (1999). 1, 2

[28] Z. Rahimi, W. Sumelka, X. J. Yang, Linear and non-linear free vibration of nano beams based on a new fractional non-local theory, Engineering Computations, 34 (2017), 1754-1770. 1

[29] K. M. Saad, E. H. AL-Shareef, M. S. Mohamed, X. J. Yang, Optimal q-homotopy analysis method for time-space fractional gas dynamics equation, D. Eur. Phys. J. Plus, 132 (2017), 23 pages. 1

[30] O. Sharomi, A. B. Gumel, Curtailing smoking dynamics: a mathematical modeling approach, Appl. Math. Comput., 195 (2008), 475-499. 1

[31] J. Singh, D. Kumar, M. Al Qurashi, D. Baleanu, A new fractional model for giving up smoking dynamics, Adv. Difference Equ., 2017 (2017), 16 pages. 1, 1, 3.1

[32] J. Singh, D. Kumar, Z. Hammouch, A. Atangana, A fractional epidemiological model for computer viruses pertaining to a new fractional derivative, Appl. Math. Comput., 316 (2018), 504-515. 1

[33] Y. Sun, B. Indraratna, J. P. Carter, T. Marchant, S. Nimbalkar, Application of fractional calculus in modelling ballast deformation under cyclic loading, Comput. Geotech., 82, (2017), 16-30. 1

[34] A. Yadav, P. K. Srivastava, A. Kumar, Mathematical model for smoking: Effect of determination and education, Int. J. Biomath., 8 (2015), 14 pages. 1

[35] G. Zaman, Qualitative behavior of giving up smoking models, Bull. Malays. Math. Sci. Soc., 34 (2011), 12 pages. 1

[36] A. Zeb, F. Bibi, G. Zaman, Optimal control strategies in square root dynamics of smoking model, International Journal of Scientific World, 3 (2015), 91-97. 1

[37] A. Zeb, G. Zaman, V. S. Erturk, B. Alzalg, F. Yousafzai, M. Khan, Approximating a Giving Up Smoking Dynamic on Adolescent Nicotine Dependence in Fractional Order, PloS one, 11 (2016), 10 pages. 1

[38] Y. D. Zhang, S. H. Wang, J.-F. Yang, Z. Zhang, P. Phillips, P. Sun, J. Yan, A Comprehensive Survey on Fractional Fourier Transform, Fund. Inform., 151 (2017), 1-48. 1 